\title{
Linearizability of Nonlinear Equations on a Quad-Graph by a Point, Two Points and Generalized Hopf-Cole Transformations ${ }^{\star}$
}

Decio LEVI ${ }^{\dagger}$ and Christian SCIMITERNA

${ }^{\dagger}$ Dipartimento di Ingegneria Elettronica, Università degli Studi Roma Tre and Sezione INFN, Roma Tre, Via della Vasca Navale 84, 00146 Roma, Italy

E-mail: levi@roma3.infn.it

URL: http://optow.ele.uniroma3.it/levi.html

$¥$ Dipartimento di Fisica, Dipartimento di Ingegneria Elettronica, Università degli Studi Roma Tre and Sezione INFN, Roma Tre, Via della Vasca Navale 84, 00146 Roma, Italy

E-mail: scimiterna@fis.uniroma3.it

Received April 15, 2011, in final form August 11, 2011; Published online August 18, 2011

http://dx.doi.org/10.3842/SIGMA.2011.079

Abstract. In this paper we propose some linearizability tests of partial difference equations on a quad-graph given by one point, two points and generalized Hopf-Cole transformations. We apply the so obtained tests to a set of nontrivial examples.

Key words: quad-graph equations; linearizability; point transformations; Hopf-Cole transformations

2010 Mathematics Subject Classification: 39A14

\section{Introduction}

In [3] one has provided necessary conditions for the linearizability of a vast class of real dispersive multilinear difference equations on a quad-graph (see Fig. 1). These conditions, obtained by

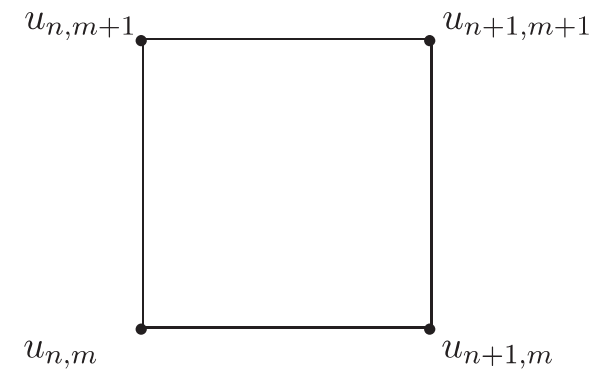

Figure 1. The quad-graph where a partial difference equation is defined.

considering the multiscale expansion up to fifth order in the perturbation parameter, are not sufficient to fix all the free parameters of the equation and one needs to make some further ansatz or to use other techniques to fix it.

So to verify these results and provide alternative ways to prove linearizability of partial difference equations on a quad-graph we write down here a set of algorithmic conditions obtained

${ }^{\star}$ This paper is a contribution to the Proceedings of the Conference "Symmetries and Integrability of Difference Equations (SIDE-9)" (June 14-18, 2010, Varna, Bulgaria). The full collection is available at http://www.emis.de/journals/SIGMA/SIDE-9.html 
by postulating the existence of linearizing transformations, i.e. classes of transformations which reduce a given partial difference equation on a quad-graph

$$
\mathcal{E}=\mathcal{E}\left(u_{n, m}, u_{n, m+1}, u_{n+1, m}, u_{n+1, m+1}\right)=0, \quad \frac{\partial \mathcal{E}}{\partial u_{n+i, m+j}} \neq 0, \quad i, j=0,1,
$$

for a field $u_{n, m}$ into a linear autonomous equation for $\tilde{u}_{n, m}$

$$
\tilde{u}_{n, m}+a \tilde{u}_{n+1, m}+b \tilde{u}_{n, m+1}+c \tilde{u}_{n+1, m+1}=0
$$

where $a, b$ and $c$ are some $(n, m)$-independent arbitrary nonzero constants. The choice that (1.2) be autonomous is a severe restriction but is a natural simplifying ansatz when one is dealing with autonomous equations. Moreover, as (1.1), (1.2) are taken to be autonomous equations, i.e. they have no $n, m$ dependent coefficients, they are translationally invariant under shifts in $n$ and $m$. So we can with no loss of generality choose as reference point $n=0$ and $m=0$. This will also be assumed to be true for all the tranformations we will study in the following. We will consider here one, two points and Hopf-Cole transformations.

By a one point transformation we mean a transformation

$$
\tilde{u}_{0,0}=f\left(u_{0,0}\right)
$$

between (1.2) and (1.1) characterized by a function depending just from the function $u_{0,0}$ and maybe on some constant parameters. It will be a Lie point transformation if $f=f_{0,0}$ satisfies all Lie group axioms. In the following we will only assume the differentiability of the function $f$ up to at least second order.

A natural generalization is when one considers two points transformations

$$
\tilde{u}_{0,0}=g\left(u_{0,0}, u_{0,1}\right) \text {, }
$$

characterized by a function $g=g_{0,0}$ depending on two lattice points, $u_{0,0}$ and $u_{0,1}$. The alternative choice when $g=g\left(u_{0,0}, u_{1,0}\right)$ will be studied elsewhere. Two lattice points is the minimum number of points necessary to provide in the continuous limit the first derivative and contact symmetries have been introduced by Lie as symmetries depending on first derivatives. Often contact symmetries are also called Miura transformations [10] as R. Miura introduced them to transform the KdV into the MKdV equation and have played a very important role in the integrability of the KdV equation. Equation (1.4) contains the transformation (1.3) as a subcase but here we will assume $\frac{\partial g}{\partial u_{0,1}} \neq 0$. Under this hypothesis the conditions for point transformations are not obtained as a limiting case of the ones for contact transformations. So one and two points transformations will be treated as independent cases.

By a generalized Hopf-Cole transformation we mean a transformation [2, 5]

$$
\begin{aligned}
& \tilde{u}_{0,1}=h\left(u_{0,0}, u_{0,1}\right) \tilde{u}_{0,0}, \\
& \tilde{u}_{1,0}=k\left(u_{0,0}, u_{1,0}, u_{0,1}, u_{1,1}\right), \tilde{u}_{0,0}
\end{aligned}
$$

where $k$ is a scalar function given in term of $h$ by

$$
k \doteq-\frac{1+b h_{0,0}\left(u_{0,0}, u_{0,1}\right)}{a+c h_{1,0}\left(u_{1,0}, u_{1,1}\right)} .
$$

The compatibility between (1.5) and (1.6) is to be identically satisfied on the nonlinear equation (1.1) while the condition (1.7) is necessary for $\tilde{u}_{n, m}$ to satisfy the linear equation (1.2). When $h\left(u_{0,0}, u_{0,1}\right)=u_{0,0}$ and $k\left(u_{0,0}, u_{1,0}, u_{0,1}, u_{1,1}\right)=u_{1,0} u_{0,0}-2 u_{0,0}$ then $\tilde{u}_{0,0}$ will satisfy a linear discrete heat equation and $u_{0,0}$ a discrete Burgers equation [6]. In such a case (1.5) is the discrete equivalent of the Hopf-Cole transformation which linearize the Burgers equation. 
In Section 2 we discuss point transformations, present the integrability conditions which ensure that the given equation is a $C$-integrable equation and the differential equations which define the function $f$. In a similar way in Section 3 we analyze two points transformations. In Section 4 we present the conditions which ensure that the given equation is a $C$-integrable equation and the differential equations which define the function $h$ for the Hopf-Cole transformation. In Section 5 we shall present a few examples while in the final section we present some conclusive remarks and open problems.

\section{Linearization by a point transformation}

In this section we discuss point transformations as, being defined by a function of just one variable, they are the simplest transformation we can propose. We will state in detail the procedure used, which will be applied later in all the other cases. This procedure follows a similar one introduced in the case of the analysis of formal symmetries for integrable quad-graph equations $[7,8]$. In particular we describe how we get from one side the determining equations which give the transformation and on the other side the conditions under which the equation (1.1) might be linearizable. The latter are necessary conditions which the given equation has to satisfy if a point transformation which linearizes the equation exists. If the conditions are satisfied then we can solve the partial differential equation determining the transformation and get a first approximation to the point transformation. However only if also the initial determining equation is satisfied the system is linearizable.

Assuming the existence of a point transformation (1.3) which linearizes (1.1), equation (1.2) reads

$$
f_{0,0}+a f_{1,0}+b f_{0,1}+c f_{1,1}=0 .
$$

In (2.1) and in the following equations we assume $u_{0 j}$ and $u_{i 0}$ as independent variables and consequently the variable $u_{1,1}$ appearing in the last term is not independent but it can be written in term of independent variables using the equation $(1.1)[7,9]$. To be able to do so, we assume that (1.1) is solvable with respect to $u_{1,1}$

$$
u_{1,1}=F\left(u_{0,0}, u_{0,1}, u_{1,0}\right),
$$

where, as (1.1) depends on all lattice points we must have

$$
F_{, u_{0,1}} \neq 0, \quad F_{, u_{1,0}} \neq 0, \quad F_{, u_{0,0}} \neq 0 .
$$

To solve the functional equation (2.1) we apply the Abel technique [1], i.e. we rewrite it as a differential equation. The solution of its differential consequences is a necessary condition for the functional equation to be satisfied.

Let us differentiate (2.1) with respect to $u_{0,1}$ and then apply the logarithmic function. We get:

$$
\left(T_{1}-1\right) \log \frac{d f_{0,1}}{d u_{0,1}}=\log \tilde{F},
$$

where $\tilde{F}$ is given by

$$
\tilde{F}\left(u_{0,0}, u_{0,1}, u_{1,0}\right) \doteq-\frac{b}{c F_{, u_{0,1}}} .
$$

We can always introduce a differential operator $\mathcal{A}$ such that

$$
\mathcal{A} \phi\left(u_{1,1}\right)=0,
$$


where $\phi$ is an arbitrary function of its argument. The most general operator of this form reads

$$
\mathcal{A}=\frac{\partial}{\partial u_{0,1}}+S^{(1)}\left(u_{0,1}, u_{0,0}, u_{1,0}\right) \frac{\partial}{\partial u_{1,0}}+S^{(2)}\left(u_{0,1}, u_{0,0}, u_{1,0}\right) \frac{\partial}{\partial u_{0,0}},
$$

where $S^{(i)}\left(u_{0,1}, u_{0,0}, u_{1,0}\right), i=1,2$ are arbitrary functions of their variables. Equation (2.5) is satisfied for any function $\phi$ if

$$
S^{(1)}=-\frac{F_{, u_{0,1}}+S^{(2)} F_{, u_{0,0}}}{F_{, u_{1,0}}} .
$$

There is no further condition to fix $S^{(2)}\left(u_{0,1}, u_{0,0}, u_{1,0}\right)$. Applying the operator $\mathcal{A}$ onto (2.4) we get

$$
\begin{aligned}
-\frac{d}{d u_{0,1}} \log \frac{d f_{0,1}}{d u_{0,1}} & =\mathcal{A} \log \tilde{F} \\
& =-\frac{1}{F_{, u_{1,0}} F_{, u_{0,1}}}\left(W_{\left(u_{0,1}\right)}\left[F_{, u_{1,0}} ; F_{, u_{0,1}}\right]+S^{(2)} W_{\left(u_{0,1}\right)}\left[F_{, u_{1,0}} ; F_{, u_{0,0}}\right]\right),
\end{aligned}
$$

which is a differential equation for $f_{0,1}\left(u_{0,1}\right)$. In $(2.6) W_{(x)}[f ; g]$ is the Wronskian operator with respect to the variable $x$ of the functions $f(x)$ and $g(x)$, defined as

$$
W_{(x)}[f ; g] \doteq f g_{, x}-g f_{, x}
$$

The left hand side of (2.6) depends only on $u_{0,1}$ while the right hand side depends on $u_{0,0}, u_{1,0}$ and $u_{0,1}$ through the given nonlinear difference equation and the up to now arbitrary function $S^{(2)}\left(u_{0,1}, u_{0,0}, u_{1,0}\right)$. So we must have

$$
\frac{\partial}{\partial u_{0,0}} \mathcal{A} \log \tilde{F}=0, \quad \frac{\partial}{\partial u_{1,0}} \mathcal{A} \log \tilde{F}=0,
$$

i.e.

$$
\begin{aligned}
& W_{\left(u_{0,1}\right)}\left[F_{, u_{1,0}} ; F_{, u_{0,0}}\right] S_{, u_{0}, 0}^{(2)}+\mathcal{K}^{(1)} S^{(2)}+\mathcal{K}^{(0)}=0, \\
& W_{\left(u_{0,1}\right)}\left[F_{, u_{1,0}} ; F_{, u_{0,0}}\right] S_{,_{1,0}}^{(2)}+\mathcal{H}^{(1)} S^{(2)}+\mathcal{H}^{(0)}=0,
\end{aligned}
$$

with

$$
\begin{aligned}
& \mathcal{K}^{(0)}\left(u_{0,0}, u_{1,0}, u_{0,1}\right) \doteq \frac{W_{\left(u_{0,0}\right)}\left[F_{, u_{1,0}} F_{, u_{0,1}} ; W_{\left(u_{0,1}\right)}\left[F_{, u_{1,0}} ; F_{, u_{0,1}}\right]\right]}{F_{, u_{1,0}} F_{u_{0,1}}}, \\
& \mathcal{K}^{(1)}\left(u_{0,0}, u_{1,0}, u_{0,1}\right) \doteq \frac{W_{\left(u_{0,0}\right)}\left[F_{, u_{1,0}} F_{, u_{0,1}} ; W_{\left(u_{0,1}\right)}\left[F_{, u_{1,0}} ; F_{, u_{0,0}}\right]\right]}{F_{, u_{1,0}} F_{, u_{0,1}}} \\
& \mathcal{H}^{(0)}\left(u_{0,0}, u_{1,0}, u_{0,1}\right) \doteq \frac{W_{\left(u_{1,0}\right)}\left[F_{, u_{1,0}} F_{, u_{0,1}} ; W_{\left(u_{0,1}\right)}\left[F_{, u_{1,0}} ; F_{, u_{0,1}}\right]\right]}{F_{, u_{1,0}} F_{, u_{0,1}}}, \\
& \mathcal{H}^{(1)}\left(u_{0,0}, u_{1,0}, u_{0,1}\right) \doteq \frac{W_{\left(u_{1,0}\right)}\left[F_{, u_{1,0}} F_{, u_{0,1}} ; W_{\left(u_{0,1}\right)}\left[F_{, u_{1,0}} ; F_{, u_{0,0}}\right]\right]}{F_{, u_{1,0}} F_{, u_{0,1}}} .
\end{aligned}
$$

As $(2.7),(2.8)$ must be valid for any given function $S^{(2)}\left(u_{0,1}, u_{0,0}, u_{1,0}\right)$, it follows that

$$
W_{\left(u_{0,1}\right)}\left[F_{, u_{1,0}} ; F_{, u_{0,0}}\right]=0
$$

and

$$
\mathcal{K}^{(0)}=0, \quad \mathcal{H}^{(0)}=0 .
$$


When (2.9), (2.10) are satisfied also $\mathcal{K}^{(1)}$ and $\mathcal{H}^{(1)}$ are null. Equations (2.9), (2.10) are necessary conditions for the linearizability by point transformations. If (1.1) is multilinear, the condition $\mathcal{H}^{(0)}=0$ is always identically satisfied.

In conclusion, we can state the following theorems:

Theorem 1. Given a partial difference equation (1.1) on a quad-graph, there exists a linearizing point transformation only if (2.9) and (2.10) are satisfied.

Theorem 2. Given a partial difference equation (1.1) on a quad-graph, if Theorem 1 is satisfied, then (2.6) will provide the possible point transformation up to an integration constant. If the obtained $f\left(u_{0,0}\right)$ verifies (2.1) then the transformation (1.3) linearizes our partial difference equation.

\section{Two points transformations}

Introducing the two points transformation (1.4) into the linear equation (1.2) we get

$$
g_{0,0}\left(u_{0,0}, u_{0,1}\right)+a g_{1,0}\left(u_{1,0}, u_{1,1}\right)+b g_{0,1}\left(u_{0,1}, u_{0,2}\right)+c g_{1,1}\left(u_{1,1}, u_{1,2}\right)=0
$$

an equation which has to be identically satisfied for any value of the independent variables of our problem. In (3.1), apart from the independent functions $u_{0,0}, u_{1,0}, u_{0,2}$ and $u_{0,1}$, there appear the functions $u_{1,1}$ and $u_{1,2}$. The function $u_{1,1}$ is expressed in term of the independent variables taking into account the partial difference quad-graph equation (1.1). An equation involving $u_{1,2}$ is obtained from (1.1) by shifting the second index by 1, i.e. considering the partial difference quad-graph equation $\mathcal{E}\left(u_{0,1}, u_{0,2}, u_{1,1}, u_{1,2}\right)=0$. However this equation does not express directly $u_{1,2}$ in terms of the independent variables but it involves $u_{1,1}$ which is expressed in term of the independent variables through the partial difference quad-graph equation itself. This fact complicates the equations obtained for the differential consequences of (3.1). To avoid it we have two possibilities, either back-shifting (1.2) once with respect to the second index, i.e. considering, in place of (3.1), the following equation

$$
g_{0,-1}\left(u_{0,-1}, u_{0,0}\right)+a g_{1,-1}\left(u_{1,-1}, u_{1,0}\right)+b g_{0,0}\left(u_{0,0}, u_{0,1}\right)+c g_{1,0}\left(u_{1,0}, u_{1,1}\right)=0
$$

or, back-shifting once with respect to the first index and once with respect to the second, i.e.

$$
g_{-1,-1}\left(u_{-1,-1}, u_{-1,0}\right)+a g_{0,-1}\left(u_{0,-1}, u_{0,0}\right)+b g_{-1,0}\left(u_{-1,0}, u_{-1,1}\right)+c g_{0,0}\left(u_{0,0}, u_{0,1}\right)=0 .
$$

In (3.2), (3.3) the variables $u_{-1,-1}, u_{-1,1}, u_{1,-1}$ and $u_{1,1}$ are not independent and from (1.1) we have, apart from (2.2)

$$
\begin{aligned}
& u_{-1,1}=G\left(u_{-1,0}, u_{0,0}, u_{0,1}\right), \quad u_{-1,-1}=H\left(u_{0,0}, u_{-1,0}, u_{0,-1}\right), \\
& u_{1,-1}=K\left(u_{0,-1}, u_{0,0}, u_{1,0}\right) .
\end{aligned}
$$

As (1.1) depends on all lattice points we must have, apart from (2.3)

$$
\begin{aligned}
& G_{, u_{0,1}} \neq 0, \quad G_{, u_{-1,0}} \neq 0, \quad G_{, u_{0,0}} \neq 0, \\
& H_{, u_{0,-1}} \neq 0, \quad H_{u_{-1,0}} \neq 0, \quad H_{, u_{0,0}} \neq 0 \text {, } \\
& K_{, u_{0,-1}} \neq 0, \quad K_{, u_{1,0}} \neq 0, \quad K_{, u_{0,0}} \neq 0 .
\end{aligned}
$$

Moreover, introducing the operators $T_{1}$ and $T_{2}$ such that $T_{1} f_{0,0}=f_{1,0}, T_{2} f_{0,0}=f_{0,1}$, we have the following relations between the derivatives of the functions $F, G, H$ and $K$ :

$$
\left.F_{, u_{0,1}}\left[T_{1} G\right]_{, u_{1,1}}\right|_{u_{1,1}=F}=1,\left.\quad F_{, u_{1,0}}\left[T_{2} K\right]_{, u_{1,1}}\right|_{u_{1,1}=F}=1,
$$




$$
\left.K_{, u_{0,-1}}\left[T_{1} H\right]_{, u_{1,-1}}\right|_{u_{1,-1}=K}=1,\left.\quad G_{, u_{-1,0}}\left[T_{2} H\right]_{, u_{-1,1}}\right|_{u_{-1,1}=G}=1 .
$$

Taking into account (3.4) it turns out that (3.2) and (3.3) give the same necessary conditions. So it is sufficient to consider one of them, say (3.2).

Differentiating (3.2) once with respect to $u_{0,1}$, we get

$$
b \frac{\partial g_{0,0}}{\partial u_{0,1}}\left(u_{0,0}, u_{0,1}\right)+c \frac{\partial g_{1,0}}{\partial u_{1,1}}\left(u_{1,0}, u_{1,1}\right) \frac{\partial F}{\partial u_{0,1}}\left(u_{0,0}, u_{0,1}, u_{1,0}\right)=0 .
$$

Applying the logarithmic function to (3.5) we get the differential difference equation for the function $g$

$$
\left(T_{1}-1\right) \log \frac{\partial g_{0,0}}{\partial u_{0,1}}=\log \tilde{F}, \quad \tilde{F}\left(u_{0,0}, u_{0,1}, u_{1,0}\right) \doteq-\frac{1}{\frac{c}{b} \frac{\partial F}{\partial u_{0,1}}},
$$

where $\tilde{F}$ is an explicit function given in term of the given quad-graph partial difference equation $\mathcal{E}$. A solution of (3.6) could be obtained by summing it up. However in this case the resulting solution $g$ would not be of the required form (1.4).

So, to find a solution of (3.6) of the required form, we simplify (3.6) by introducing a differential operator $\mathcal{A}$ such that

$$
\mathcal{A} \phi\left(u_{1,0}, u_{1,1}\right)=0
$$

where $\phi$ is an arbitrary function of its arguments. The most general operator of this form reads

$$
\mathcal{A}=\frac{\partial}{\partial u_{0,1}}+S_{a}^{(1)}\left(u_{0,0}, u_{0,1}, u_{1,0}\right) \frac{\partial}{\partial u_{0,0}}+S_{a}^{(2)}\left(u_{0,0}, u_{0,1}, u_{1,0}\right) \frac{\partial}{\partial u_{1,0}},
$$

where $S_{a}^{(i)}\left(u_{0,0}, u_{0,1}, u_{1,0}\right), i=1,2$ are arbitrary functions of the independent variables to be determined. Equation (3.7) is satisfied for any function $\phi$ if

$$
S_{a}^{(1)}=-\frac{F_{, u_{0,1}}}{F_{, u_{0,0}}}, \quad S_{a}^{(2)}=0 .
$$

Applying the operator $\mathcal{A}$ onto (3.6) and defining $\psi\left(u_{0,0}, u_{0,1}\right) \doteq \log \frac{\partial g_{0,0}}{\partial u_{0,1}}$, we get

$$
\psi_{, u_{0,1}}-\frac{F_{, u_{0,1}}}{F_{, u_{0,0}}} \psi_{, u_{0,0}}=\mathcal{R}\left(u_{0,0}, u_{0,1}, u_{1,0}\right) \doteq \frac{1}{F_{, u_{0}, 0} F_{, u_{0,1}}} W_{\left(u_{0,1}\right)}\left[F_{, u_{0}, 0} ; F_{, u_{0,1}}\right] .
$$

Equation (3.10) is a differential equation for the function $\psi\left(u_{0,0}, u_{0,1}\right)$, i.e. for the function characterizing the two points transformation whose coefficients depend on the given quad-graph partial difference equation $\mathcal{E}$. In (3.10) the function $\psi$ depends just on $u_{0,0}, u_{0,1}$ while the terms depending on the given quad-graph partial difference equation $\mathcal{E}$ depend on $u_{0,0}, u_{0,1}, u_{1,0}$. As the quad-graph equation $\mathcal{E}$ depends also on the variable $u_{1,0},(3.10)$ will be an equation determining the two points transformation only if some further compatibility conditions are satisfied.

Differentiating (3.10) once with respect to $u_{1,0}$, we get the following alternatives:

1. If $W_{\left(u_{1,0}\right)}\left[F_{, u_{0,1}} ; F_{, u_{0,0}}\right]=0$ identically, we must have

$$
\frac{\partial}{\partial u_{1,0}} \mathcal{R}=0
$$

which is a necessary condition for the linearizability of (1.1) through the two points transformation (1.4). 
2. If $W_{\left(u_{1,0}\right)}\left[F_{, u_{0,1}} ; F_{, u_{0,0}}\right] \neq 0$, we get

$$
\psi_{, u_{0,0}}=\mathcal{M}\left(u_{0,0}, u_{0,1}, u_{1,0}\right), \quad \mathcal{M} \doteq \frac{F_{, u_{0,0}}^{2} \mathcal{R}_{, u_{1,0}}}{W_{\left(u_{1,0}\right)}\left[F_{, u_{0,1}} ; F_{, u_{0,0}}\right]} .
$$

Inserting (3.12) in (3.10), we get

$$
\psi_{, u_{0,1}}=\mathcal{N}\left(u_{0,0}, u_{0,1}, u_{1,0}\right),
$$

where

$$
\mathcal{N} \doteq \frac{F_{, u_{0,1}}}{F_{u_{0,0}}} \mathcal{M}+\mathcal{R}
$$

As the left hand side of (3.12) is independent of $u_{1,0}$, we get the necessary condition

$$
\frac{\partial}{\partial u_{1,0}} \mathcal{M}=0
$$

It is straightforward to prove that, if (3.14) is satisfied, also $\frac{\partial}{\partial u_{1,0}} \mathcal{N}=0$ will be true. Moreover the compatibility of (3.12), (3.13) gives another necessary condition

$$
\frac{\partial}{\partial u_{0,1}} \mathcal{M}=\frac{\partial}{\partial u_{0,0}} \mathcal{N}
$$

We can differentiate (3.2) with respect to $u_{0,-1}$. In this case we get

$$
\left(T_{1}-1\right) \log \frac{\partial g_{0,-1}}{\partial u_{0,-1}}=\log \tilde{K}, \quad \tilde{K}\left(u_{0,0}, u_{1,0}, u_{0,-1}\right) \doteq-\frac{1}{a \frac{\partial K}{\partial u_{0,-1}}} .
$$

We can introduce the differential operator $\mathcal{B}$

$$
\mathcal{B}=\frac{\partial}{\partial u_{0,-1}}+S_{b}^{(1)}\left(u_{0,0}, u_{1,0}, u_{0,-1}\right) \frac{\partial}{\partial u_{0,0}}+S_{b}^{(2)}\left(u_{0,0}, u_{1,0}, u_{0,-1}\right) \frac{\partial}{\partial u_{1,0}},
$$

such that

$$
\mathcal{B} \xi\left(u_{1,0}, u_{1,-1}\right)=0,
$$

where $\xi$ is an arbitrary function of its arguments. As (3.18) has to be satisfied for any $\xi$, we get

$$
S_{b}^{(1)}=-\frac{K_{, u_{0,-1}}}{K_{, u_{0,0}}}, \quad S_{b}^{(2)}=0
$$

Applying the operator $\mathcal{B}$ onto (3.16) and defining $\phi\left(u_{0,-1}, u_{0,0}\right) \doteq \log \frac{\partial g_{0,-1}}{\partial u_{0,-1}}$ we get the following differential equation for the function $\phi$

$$
\phi_{, u_{0,-1}}-\frac{K_{, u_{0,-1}}}{K_{, u_{0,0}}} \phi_{, u_{0,0}}=\mathcal{T}\left(u_{0,0}, u_{1,0}, u_{0,-1}\right) \doteq \frac{1}{K_{, u_{0,0}} K_{, u_{0,-1}}} W_{\left(u_{0,-1}\right)}\left[K_{, u_{0,0}} ; K_{, u_{0,-1}}\right] .
$$

Differentiating equation (3.19) once with respect to $u_{1,0}$, we get the following alternatives:

1. $W_{\left(u_{1,0}\right)}\left[K_{, u_{0,-1}} ; K_{, u_{0,0}}\right]=0$ identically, then we must have

$$
\frac{\partial}{\partial u_{1,0}} \mathcal{T}=0
$$

which is a necessary condition for linearizability of (1.1) through the two points transformation (1.4). 
2. If $W_{\left(u_{1,0}\right)}\left[K_{, u_{0,-1}} ; K_{, u_{0,0}}\right] \neq 0$, we get

$$
\phi_{, u_{0,0}}=\mathcal{P}\left(u_{0,0}, u_{1,0}, u_{0,-1}\right), \quad \mathcal{P} \doteq \frac{K_{, u_{0,0}}^{2} \mathcal{T}_{, u_{1,0}}}{W_{\left(u_{1,0}\right)}\left[K_{, u_{0,-1}} ; K_{, u_{0,0}}\right]} .
$$

Inserting (3.21) in (3.19), we get

$$
\phi_{, u_{0,-1}}=\mathcal{Q}\left(u_{0,0}, u_{1,0}, u_{0,-1}\right),
$$

where

$$
\mathcal{Q} \doteq \frac{K_{, u_{0,-1}}}{K_{, u_{0,0}}} \mathcal{P}+\mathcal{T}
$$

As the left hand side of (3.21) is independent of $u_{1,0}$, we get the necessary condition

$$
\frac{\partial}{\partial u_{1,0}} \mathcal{P}=0
$$

It is straightforward to prove that, if (3.23) is satisfied, also $\frac{\partial}{\partial u_{1,0}} \mathcal{Q}=0$ will be true. Moreover the compatibility of (3.21), (3.22) gives another necessary condition

$$
\frac{\partial}{\partial u_{0,-1}} \mathcal{P}=\frac{\partial}{\partial u_{0,0}} \mathcal{Q}
$$

We summarize the results so far obtained in the following theorems:

Theorem 3. Given a partial difference equation (1.1) on a quad-graph we can construct the Wronskian functions $\mathbb{F}=W_{\left(u_{1,0}\right)}\left[F_{, u_{0,1}} ; F_{, u_{0,0}}\right]$ and $\mathbb{K}=W_{\left(u_{1,0}\right)}\left[K_{, u_{0,-1}} ; K_{, u_{0,0}}\right]$. Depending on the values of $\mathbb{F}$ and $\mathbb{K}$ we have different necessary conditions for the existence of a linearizing two points transformation (1.4).

1. $\mathbb{F} \neq 0, \mathbb{K} \neq 0$. We have different results according to the value of $\mathcal{M}$.

(a) If $\mathcal{M} \neq 0$, apart from the linearizability conditions (3.14), (3.15), (3.23), (3.24) the following compatible conditions must be satisfied:

$$
\left(\log \frac{\left[T_{2} \mathcal{P}\right]}{\mathcal{M}}\right)_{, u_{0,0}}=\mathcal{M}-\left[T_{2} \mathcal{Q}\right], \quad\left(\log \frac{\left[T_{2} \mathcal{P}\right]}{\mathcal{M}}\right)_{, u_{0,1}}=\mathcal{N}-\left[T_{2} \mathcal{P}\right]
$$

(b) If $\mathcal{M}=0$, the linearizability conditions are (3.15), (3.23), (3.24).

2. $\mathbb{F} \neq 0, \mathbb{K}=0$. Apart from the linearizability conditions (3.15), (3.14), (3.20) we have different results according to the value of $\mathcal{T}_{, u_{0,0}}$.

(a) If $\mathcal{T}_{, u_{0,0}}=0$

$$
\mathcal{M}_{, u_{0,0}}=\left(\frac{\left[T_{2} K\right]_{, u_{0,0}}}{\left[T_{2} K\right]_{, u_{0,1}}} \mathcal{M}\right)_{, u_{0,1}}+\left(\frac{\left[T_{2} K\right]_{, u_{0,0}}}{\left[T_{2} K\right]_{, u_{0,1}}} \mathcal{N}-\mathcal{M}+\left[T_{2} \mathcal{T}\right]\right) \mathcal{M} .
$$

(b) If $\mathcal{T}_{u_{0,0}} \neq 0$, defining

$$
\mathcal{S} \doteq-\frac{\left(\frac{\left[T_{2} K\right]_{, u_{0,0}}}{\left[T_{2} K\right]_{, u_{0,1}}} \mathcal{M}\right)_{, u_{0,1}}+\frac{\left[T_{2} K\right]_{, u_{0}, 0}}{\left[T_{2} K\right]_{, u_{0}, 1}} \mathcal{M N}+\left[T_{2} \mathcal{T}\right] \mathcal{M}-\mathcal{M}^{2}-\mathcal{M}_{, u_{0,0}}}{\left[T_{2} \mathcal{T}\right]_{, u_{0,1}}}
$$

we get the following linearizability conditions

$$
\mathcal{S}_{, u_{0,1}}=\mathcal{M}-\mathcal{S N}, \quad \mathcal{S}_{u_{0,0}}=\frac{\left[T_{2} K\right]_{, u_{0,0}}}{\left[T_{2} K\right]_{, u_{0,1}}} \mathcal{M}+\left[T_{2} \mathcal{T}\right] \mathcal{S}-\mathcal{S M}
$$


3. $\mathbb{F}=0, \mathbb{K} \neq 0$. Apart from the linearizability conditions (3.24), (3.23), (3.11) we have different results according to the value of $\mathcal{R}_{u_{0,0}}$.

(a) If $\mathcal{R}_{, u_{0,0}}=0$,

$$
\left(\left[T_{2} \mathcal{P}\right]\right)_{, u_{0,1}}=\left(\frac{F_{, u_{0,1}}}{F_{, u_{0,0}}}\left[T_{2} \mathcal{P}\right]\right)_{, u_{0,0}}+\left(\frac{F_{, u_{0,1}}}{F_{, u_{0}, 0}}\left[T_{2} \mathcal{Q}\right]-\left[T_{2} \mathcal{P}\right]+\mathcal{R}\right)\left[T_{2} \mathcal{P}\right]
$$

(b) If $\mathcal{R}_{, u_{0,0}} \neq 0$, defining

$$
\mathcal{U} \doteq-\frac{\left(\frac{F_{, u_{0}, 1}}{F_{, u_{0}, 0}}\left[T_{2} \mathcal{P}\right]\right)_{, u_{0,0}}+\frac{F_{, u_{0,1}}}{F, u_{0,0}}\left[T_{2} \mathcal{P}\right]\left[T_{2} \mathcal{Q}\right]+\mathcal{R}\left[T_{2} \mathcal{P}\right]-\left(\left[T_{2} \mathcal{P}\right]\right)^{2}-\left(\left[T_{2} \mathcal{P}\right]\right)_{, u_{0,1}}}{\mathcal{R}_{, u_{0,0}}}
$$

we get the further linearizability conditions

$$
\mathcal{U}_{, u_{0,0}}=\left[T_{2} \mathcal{P}\right]-\mathcal{U}\left[T_{2} \mathcal{Q}\right], \quad \mathcal{U}_{u_{0,1}}=\frac{F_{, u_{0,1}}}{F_{, u_{0,0}}}\left[T_{2} \mathcal{P}\right]+\mathcal{R} \mathcal{U}-\mathcal{U}\left[T_{2} \mathcal{P}\right]
$$

4. $\mathbb{F}=0, \mathbb{K}=0$. Apart from the linearizability conditions (3.20), (3.11) we have a set of conditions for the functions $F$ and $K$ involved, depending if $\frac{\left[T_{2} K\right]_{u_{0,0}}}{\left[T_{2} K\right]_{u_{0}, 1}} \frac{F_{u_{0,1}}}{F_{u_{0}, 0}}$ is equal to 1 or not. These conditions are obtained by requiring that the overdetermined system obtained by explicitating (3.10), (3.19) in term of $g=g_{0,0}$ and possibly shifting

$$
\begin{aligned}
& g_{, u_{0,0}, u_{0,0}}-\frac{\left[T_{2} K\right]_{, u_{0,0}}}{\left[T_{2} K\right]_{, u_{0,1}}} g_{, u_{0,0}, u_{0,1}}=\left[T_{2} \mathcal{T}\right] g_{, u_{0,0}}, \\
& g_{, u_{0,1}, u_{0,1}}-\frac{F_{, u_{0,1}}}{F_{, u_{0,0}}} g_{, u_{0,0}, u_{0,1}}=\mathcal{R} g_{, u_{0,1}},
\end{aligned}
$$

be solvable for any $u_{0,0}, u_{0,1}, u_{1,0}$. These equations are easy to derive by symbolic manipulation but too long to write down. So, for the sake of clarity, we do not write them down here.

Theorem 4. Given a partial difference equation (1.1) on a quad-graph, if Theorem 3 is satisfied, depending on the values of $\mathbb{F}$ and $\mathbb{K}$, we have different partial differential equations defining the two points transformation.

1. $\mathbb{F} \neq 0, \mathbb{K} \neq 0$. We have different results according to the value of $\mathcal{M}$.

(a) If $\mathcal{M} \neq 0$ we have for $g=g_{0,0}$

$$
g_{, u_{0,0}, u_{0,0}}=\left[T_{2} \mathcal{Q}\right] g_{, u_{0,0}}, \quad g_{u_{0,1}}=\frac{\left[T_{2} \mathcal{P}\right]}{\mathcal{M}} g_{, u_{0,0}}
$$

(b) If $\mathcal{M}=0$ we have $g=g^{(0)}\left(u_{0,0}\right)+g^{(1)}\left(u_{0,1}\right)$ and

$$
g_{, u_{0,0} u_{0,0}}^{(0)}=\left[T_{2} \mathcal{Q}\right] g_{, u_{0,0}}^{(0)}, \quad g_{, u_{0,1} u_{0,1}}^{(1)}=\mathcal{N} g_{, u_{0,1}}^{(1)} \text {. }
$$

2. $\mathbb{F} \neq 0, \mathbb{K}=0$. We have different results according to the value of $\mathcal{T}_{u_{0,0}}$.

(a) If $\mathcal{T}_{, u_{0,0}}=0$ the two points transformation is obtained by solving for $g=g_{0,0}$ the compatible system of partial differential equations (3.12), (3.13) and (3.26); 
(b) If $\mathcal{T}_{u_{0,0}} \neq 0$ the two points transformation is obtained by solving the compatible partial differential equations (3.13) and

$$
g_{, u_{0,0}}=\mathcal{S} g_{, u_{0,1}} .
$$

3. $\mathbb{F}=0, \mathbb{K} \neq 0$. We have different results according to the value of $\mathcal{R}_{u_{0,0}}$.

(a) If $\mathcal{R}_{, u_{0,0}}=0$ the two points transformation is obtained by solving for $g=g_{0,0}$ the compatible system of partial differential equations (3.21), (3.22) and (3.27);

(b) If $\mathcal{R}_{, u_{0,0}} \neq 0$ the two points transformation is obtained by solving the compatible partial differential equations (3.22) and

$$
g_{, u_{0,1}}=\mathcal{U} g_{, u_{0,0}} .
$$

4. $\mathbb{F}=0, \mathbb{K}=0$ the two points transformation is obtained by solving for $g=g_{0,0}$ the compatible system of partial differential equations (3.27) and (3.26).

When we have solved the PDEs for the function $g\left(u_{0,0}, u_{0,1}\right)$ we may still have arbitrary functions or arbitrary constants. These get fixed by inserting the function $g$ into the equations (3.5), (3.16) and solving them and their consequences. At the end we need to verify that (3.1) or its shifted versions (3.2), (3.3) be satisfied.

\section{Linearization by a generalized Hopf-Cole transformation}

Let us assume the existence of a generalized Hopf-Cole transformation (1.5) which linearizes (1.1) into (1.2), where $a, b$ and $c$ are arbitrary constants and the function $h=h_{0,0}$ of its two arguments is to be determined.

The nonlinear equation (1.1) can be written just in term of $h$ and $k$ as

$$
h_{1,0} k_{0,0}=k_{0,1} h_{0,0}
$$

and in terms of $h$ alone as

$$
\left(\frac{h_{0,0}+1 / b}{h_{0,0}}\right)=\left(\frac{h_{1,0}+a / c}{h_{1,0}}\right)\left(\frac{h_{0,1}+1 / b}{h_{1,1}+a / c}\right) .
$$

If we assume $h=h\left(u_{0,0}\right)$, then, differentiating (4.2) with respect to $u_{0,1}$, we find

$$
\frac{\partial}{\partial u_{0,1}} \log \left(h_{0,1}+1 / b\right)=\frac{\partial}{\partial u_{1,1}} \log \left(h_{1,1}+a / c\right) \frac{\partial}{\partial u_{0,1}} F
$$

and, by carrying out the same kind of calculations as in Section 2, we find the same linearizability conditions (2.9), (2.10) as for point transformations, i.e. Theorem 1 will be valid. However the differential equation for the transformation is different and is given by

$$
\frac{d}{d u_{0,1}} \log \frac{d}{d u_{0,1}} \log \left(h_{0,1}+1 / b\right)=\frac{1}{F_{, u_{1,0}} F_{, u_{0,1}}} W_{\left(u_{0,1}\right)}\left[F_{, u_{1,0}} ; F_{, u_{0,1}}\right] .
$$

So, if an equation is linearizable by a point transformation it can also be linearizable by a HopfCole transformation depending on one point only. However the effective linearizing transformation is different and thus one can find a nonlinear partial difference equation on the square which is linearizable by a Hopf-Cole transformation with $h=h\left(u_{0,0}\right)$ but not by a point transformation (1.3). 
If the left hand side of (4.2) depends on $u_{0,0}$ and $u_{0,1}$, the first left term in the right hand side depends on $u_{1,0}$ and $u_{1,1}$ and the second one on $u_{0,1}, u_{0,2}, u_{1,1}$ and $u_{1,2}$. The variable $u_{1,1}$ is given in terms of the independent variables by (2.2) while $u_{1,2}$ can be rewritten in term of the independent variables as

$$
u_{1,2}=F\left(u_{0,1}, u_{0,2}, F\left(u_{0,0}, u_{0,1}, u_{1,0}\right)\right) .
$$

So, as from (4.5) the expression of $u_{1,2}$ in terms of the independent variables depends twice on the quad-graph equation (1.1), we will consider in place of (4.1) the equation,

$$
h_{1,-1} k_{0,-1}=k_{0,0} h_{0,-1} \text {. }
$$

which in terms of $h$ alone read

$$
\left(\frac{h_{0,-1}+1 / b}{h_{0,-1}}\right)=\left(\frac{h_{1,-1}+a / c}{h_{1,-1}}\right)\left(\frac{h_{0,0}+1 / b}{h_{1,0}+a / c}\right),
$$

The left hand side of (4.6) depends on $u_{0,0}$ and $u_{0,-1}$, the first left term in the right hand side depends on $u_{1,0}$ and $u_{1,-1}=K\left(u_{0,-1}, u_{1,0}, u_{0,0}\right)$ and the second one on $u_{0,1}, u_{0,0}, u_{1,1}=$ $F\left(u_{0,1}, u_{1,0}, u_{0,0}\right)$ and $u_{1,0}$. So, the term on the left hand side of (4.6) depends on $u_{0,0}$ and $u_{0,-1}$, the first left term on the right hand side depends on $u_{1,0}, u_{0,0}$ and $u_{0,-1}$ while the second one on $u_{0,1}, u_{0,0}$ and $u_{1,0}$. Thus one can see that the three terms appearing in the equation (4.6) contain no overlapping set of variables. This is a condition necessary to get out of (4.6) some differential conditions for the functions $F$ and $K$, i.e. for the equation (1.1) to be rewritable as the compatibility condition of (1.5) and (1.6).

Let us consider (4.6) and, as we have products, we reduce it to a sum of terms by applying to it the logarithmic function. Then we differentiate the resulting equation with respect to $u_{0,1}$. Only the second term on the r.h.s. of the equality depends on $u_{0,1}$ through the dependence of $h_{1,0}$ on $u_{1,1}$ and of $h_{0,0}$. So we get:

$$
\frac{\partial}{\partial u_{0,1}} \log \left(h_{0,0}+1 / b\right)=\frac{\partial}{\partial u_{1,1}} \log \left(h_{1,0}+a / c\right) \frac{\partial}{\partial u_{0,1}} F
$$

equivalent, in structure to (3.5). The term on the l.h.s. of (4.7) depends on $u_{0,0}$ and $u_{0,1}$ while the first factor on the r.h.s. depends on $u_{1,0}$ and $u_{1,1}$ and we can always introduce the differential operator $\mathcal{A}$ as given by (3.8). So, if we apply again the logarithmic function to equation (4.7) and then the operator $\mathcal{A}$ onto the resulting equation, setting $\psi\left(u_{0,0}, u_{0,1}\right) \doteq \log \frac{\partial}{\partial u_{0,1}} \log (h+1 / b)$, we get the linear differential equation (3.10). It is worthwhile to notice that, even if the differential equation is the same when expressed in term of the variable $\psi$, its expression in term of $f$ is different from the one in term of $h$.

Let us now differentiate (4.6) with respect to $u_{0,-1}$. Proceeding in an analogous way as we did before, we get

$$
\frac{\partial}{\partial u_{0,-1}} \log \left(\frac{h_{0,-1}+1 / b}{h_{0,-1}}\right)=\frac{\partial}{\partial u_{1,-1}} \log \left(\frac{h_{1,-1}+a / c}{h_{1,-1}}\right) \frac{\partial}{\partial u_{0,-1}} K .
$$

The term on the 1.h.s. of (4.8) depends on $u_{0,-1}$ and $u_{0,0}$ while the first factor on the r.h.s. depends on $u_{1,-1}$ and $u_{1,0}$. We can always introduce the differential operator $\mathcal{B}$ as given by (3.17). So if we apply again the logarithmic function to equation (4.8) and then the operator $\mathcal{B}$ onto the resulting equation, setting $\phi\left(u_{0,-1}, u_{0,0}\right) \doteq \log \frac{\partial}{\partial u_{0,-1}} \log \frac{h_{0,-1}+1 / b}{h_{0,-1}}$, we get the linear differential equation (3.19) for $\phi$. However, as before, even if the differential equation is the same when expressed in term of the variable $\phi$, its expression in term of $f$ is different from the one in term of $h$. 
As the determining equations in terms of $\psi$ and $\phi$ are exactly the same as those of contact transformations, the linearizability conditions are as presented in Theorem 3. However the function $\psi$ and $\phi$ are defined here differently then in the case of two points transformations. So the equations defining $h=h_{0,0}$ are different. In particular (3.26) and (3.27) in this case have to be replaced by the nonlinear equations

$$
\begin{aligned}
& \frac{h_{u_{0,1} u_{0,1}}}{h+1 / b}-\left(\frac{h_{u_{0,1}}}{h+1 / b}\right)^{2}-\frac{F_{u_{0,1}}}{F_{u_{0,0}}}\left(\frac{h_{u_{0,1} u_{0,0}}}{h+1 / b}-\frac{h_{u_{0,1}} h_{u_{0,0}}}{(h+1 / b)^{2}}\right)=\mathcal{R}, \\
& {\left[\left(\frac{h_{0,-1, u_{0,-1}}}{h_{0,-1}\left(h_{0,-1}+1 / b\right)}\right)_{u_{0,-1}}-\frac{K_{u_{0,-1}}}{K_{u_{0,0}}}\left(\frac{h_{0,-1, u_{0,-1}}}{h_{0,-1}\left(h_{0,-1}+1 / b\right)}\right)_{u_{0,0}}\right] \frac{h_{0,-1}\left(h_{0,-1}+1 / b\right)}{h_{0,-1, u_{0,-1}}}=\mathcal{T} .}
\end{aligned}
$$

A simpler and sometimes more useful nonlinear equation for $h_{0,0}$ can be obtained in the following way. Let us shift (4.8) by $T_{2}$. In such a way we get

$$
\frac{\partial}{\partial u_{0,0}} \log \left(\frac{h_{0,0}+1 / b}{h_{0,0}}\right)=\frac{\partial}{\partial u_{1,0}} \log \left(\frac{h_{1,0}+a / c}{h_{1,0}}\right) \frac{\partial}{\partial u_{0,0}}\left[T_{2} K\right] .
$$

Then from $(4.7),(4.9)$ we extract the partial derivatives of $h_{0,0}$ with respect to $u_{0,0}$ and $u_{0,1}$,

$$
\begin{aligned}
h_{0,0, u_{0,1}} & =\frac{h_{0,0}+1 / b}{h_{1,0}+a / c} h_{1,0, u_{1,1}} \frac{\partial F}{\partial u_{0,1}}, \\
h_{0,0, u_{0,0}} & =\frac{a / c h_{0,0}}{1 / b h_{1,0}} \frac{h_{0,0}+1 / b}{h_{1,0}+a / c} h_{1,0, u_{1,0}} \frac{\partial\left[T_{2} K\right]}{\partial u_{0,0}} .
\end{aligned}
$$

Dividing (4.10) by (4.11) we get the following equation:

$$
\left(T_{1}-1\right) \log \frac{h_{0,0} h_{0,0, u_{0,1}}}{h_{0,0, u_{0,0}}}+\log \frac{1 / b \frac{\partial F}{\partial u_{0,1}}}{a / c \frac{\partial\left[T_{2} K\right]}{\partial u_{0,0}}}=0 .
$$

Differentiating (4.12) with respect to $u_{1,0}$ we obtain a second order nonlinear differential equation for the function $\chi\left(u_{0,0}, u_{0,1}\right)=\log \left(h_{0,0}\right)$. We have:

$$
\left[\chi_{, u_{0,0}}+\frac{\chi_{, u_{0,0}, u_{0,1}}}{\chi_{, u_{0,1}}}-\frac{\chi_{, u_{0,0}, u_{0,0}}}{\chi_{, u_{0,0}}}\right]+\mathcal{C}\left[\chi_{, u_{0,1}}+\frac{\chi_{, u_{0,1}, u_{0,1}}}{\chi_{, u_{0,1}}}-\frac{\chi_{, u_{0,1}, u_{0,0}}}{\chi_{, u_{0,0}}}\right]+\mathcal{D}=0,
$$

where

$$
\begin{aligned}
& \mathcal{C}\left(u_{-1,0}, u_{0,0}, u_{0,1}\right) \doteq\left\{T_{1}^{-1}\left[\frac{\partial F}{\partial u_{1,0}}\right]\right\}_{u_{-1,1} \rightarrow G\left(u_{-1,0}, u_{0,0}, u_{0,1}\right)}, \\
& \mathcal{D}\left(u_{-1,0}, u_{0,0}, u_{0,1}\right) \doteq\left\{T_{1}^{-1}\left[\frac{\partial}{\partial u_{1,0}} \log \left(\frac{1}{\mathcal{H}} \frac{\partial F}{\partial u_{0,1}}\right)\right]\right\}_{u_{-1,1} \rightarrow G\left(u_{-1,0}, u_{0,0}, u_{0,1}\right)}, \\
& \mathcal{H}\left(u_{0,0}, u_{1,0}, u_{0,1}\right) \doteq\left\{T_{2}\left[\frac{\partial K}{\partial u_{0,-1}}\right]\right\}_{u_{1,1} \rightarrow F\left(u_{0,0}, u_{1,0}, u_{0,1}\right)} .
\end{aligned}
$$

The first and second terms of (4.13) depend on derivatives of the unknown function $\chi\left(u_{0,0}, u_{0,1}\right)$ but the coefficient of the second term and the last one may contain also $u_{-1,0}$. So we have a further set of linearizability conditions. If $\frac{\partial}{\partial u_{-1,0}} \mathcal{C}=0$, differentiating (4.13) with respect to $u_{-1,0}$ we have

$$
\frac{\partial}{\partial u_{-1,0}} \mathcal{D}=0
$$


while, if $\frac{\partial}{\partial u_{-1,0}} \mathcal{C} \neq 0$, after a differentiation with respect to $u_{-1,0}$, we have

$$
W_{\left(u_{-1,0}\right)}\left[\mathcal{C}_{, u_{-1,0}} ; \mathcal{D}_{, u_{-1,0}}\right]=0 .
$$

In the first case, the solutions of (4.13) provides us with an ansatz of the function $h$, otherwise the function $h$ is obtained by solving the following overdetermined system of nonlinear partial differential equations

$$
\begin{aligned}
& \chi_{, u_{0,0}}=\frac{\chi_{, u_{0,0}, u_{0,0}}}{\chi_{, u_{0,0}}}-\frac{\chi_{, u_{0,0}, u_{0,1}}}{\chi_{, u_{0,1}}}+\frac{W_{\left(u_{-1,0}\right)}[\mathcal{C} ; \mathcal{D}]}{\mathcal{C}_{, u_{-1,0}}}, \\
& \chi_{, u_{0,1}}=\frac{\chi_{, u_{0,1}, u_{0,0}}}{\chi_{, u_{0,0}}}-\frac{\chi_{, u_{0,1}, u_{0,1}}}{\chi_{, u_{0,1}}}-\frac{\mathcal{D}_{, u_{-1,0}}}{\mathcal{C}_{, u_{-1,0}}} .
\end{aligned}
$$

If the condition (4.14) is satisfied, then $\frac{\partial}{\partial u_{-1,0}} \frac{W_{\left(u_{-1,0}\right)}[\mathcal{C} ; \mathcal{D}]}{\mathcal{C}_{, u_{-1,0}}}=0$. The overdetermined system $(4.15)$ is compatible iff

$$
W_{\left(u_{0,1}\right)}\left[W_{\left(u_{-1,0}\right)}[\mathcal{C} ; \mathcal{D}] ; \mathcal{C}_{, u_{-1,0}}\right]=W_{\left(u_{0,0}\right)}\left[\mathcal{C}_{, u_{-1,0}} ; \mathcal{D}_{, u_{-1,0}}\right]
$$

a further linearizability condition. Equations (4.13), (4.15) are a nonlinear partial differential system which, introducing the function

$$
\theta\left(u_{0,0}, u_{0,1}\right) \doteq \chi+\log \left(\frac{\chi_{, u_{0,1}}}{\chi_{, u_{0,0}}}\right)
$$

can be linearized and read:

$$
\begin{aligned}
& \theta_{, u_{0,0}}+\mathcal{C} \theta_{, u_{0,1}}+\mathcal{D}=0, \\
& \theta_{, u_{0,0}}=\frac{W_{\left(u_{-1,0}\right)}[\mathcal{C} ; \mathcal{D}]}{\mathcal{C}_{, u_{-1}, 0}}, \quad \theta_{, u_{0,1}}=-\frac{\mathcal{D}_{, u_{-1,0}}}{\mathcal{C}_{, u_{-1,0}}} .
\end{aligned}
$$

Once the solution of the equations (4.18) or (4.19) has been obtained, the function $h$ can be reconstructed. Starting from the definition (4.17) we get

$$
e^{\chi} \frac{\partial}{\partial u_{0,1}} e^{\chi}=e^{\theta} \frac{\partial}{\partial u_{0,0}} e^{\chi}
$$

or in terms of $h$

$$
h_{, u_{0,0}}=e^{-\theta} h h_{, u_{0,1}},
$$

which is a Hopf-like equation whose solution can be obtained for example by separation of variables. Once we have a solution, we can introduce it into the lowest order differential equations and define the arbitrary functions or constant involved. The so obtained function $h$ will provide us with a linearizing generalized Hopf-Cole transformation if the difference relation (4.2) is satisfied. Equation (4.20) can be introduced in (4.10), (4.11) and after some manipulations and the application of the operator $\mathcal{A}$ defined in (3.8), (3.9), we obtain a linear evolution equation for the function $\theta\left(u_{0,0}, u_{0,1}\right)$

$$
\theta_{, u_{0,1}}-\frac{F_{, u_{0,1}}}{F_{, u_{0,0}}} \theta_{u_{0,0}}=\tilde{\mathcal{T}}\left(u_{0,0}, u_{1,0}, u_{0,1}\right) \doteq \mathcal{A} \log \left(\frac{1}{\mathcal{H}} F_{, u_{0,1}}\right)
$$

Differentiating equation (4.21) once with respect to $u_{1,0}$, we get the following alternatives: 
1. $\mathbb{F}=0$ identically, then we must have

$$
\tilde{\mathcal{T}}_{u_{1,0}}=0,
$$

which is a necessary condition for linearizability through the Hopf-Cole transformation (1.5), (1.6), (1.7).

2. If $\mathbb{F} \neq 0$, we get

$$
\theta_{, u_{0,0}}=\tilde{\mathcal{P}}\left(u_{0,0}, u_{1,0}, u_{0,1}\right), \quad \tilde{\mathcal{P}} \doteq \frac{F_{, u_{0,0}}^{2} \tilde{\mathcal{T}}_{, u_{1,0}}}{\mathbb{F}} .
$$

Inserting (4.22) in (4.21), we get

$$
\theta_{, u_{0,1}}=\tilde{\mathcal{Q}}\left(u_{0,0}, u_{1,0}, u_{0,1}\right)
$$

where

$$
\tilde{\mathcal{Q}} \doteq \frac{F_{, u_{0,1}}}{F_{, u_{0,0}}} \tilde{\mathcal{P}}+\tilde{\mathcal{T}}
$$

As the left hand side of (4.22) is independent of $u_{1,0}$, we get the necessary condition

$$
\frac{\partial}{\partial u_{1,0}} \tilde{\mathcal{P}}=0
$$

It is straightforward to prove that, if (4.24) is satisfied, also $\frac{\partial}{\partial u_{1,0}} \tilde{\mathcal{Q}}=0$ will be true. Moreover the compatibility of (4.22), (4.23) gives another necessary condition

$$
\frac{\partial}{\partial u_{0,1}} \tilde{\mathcal{P}}=\frac{\partial}{\partial u_{0,0}} \tilde{\mathcal{Q}}
$$

As in the case of contact transformations, the combination of the two cases defined by (4.18) or (4.19) and the two cases defined by (4.21) or (4.22), (4.23) gives a total of four subcases for the specification of the function $\theta$. If the conditions (4.14), (4.16) are satisfied, the solution of the two equations (4.19) is given by

$$
\theta=\int_{u_{0,0}^{\prime}=\alpha}^{u_{0,0}^{\prime}=u_{0,0}} \frac{W_{\left(u_{-1,0}\right)}[\mathcal{C} ; \mathcal{D}]}{\mathcal{C}_{, u_{-1,0}}}\left(u_{0,0}^{\prime}, u_{0,1}\right) d u_{0,0}^{\prime}-\int_{u_{0,1}^{\prime}=\beta}^{u_{0,1}^{\prime}=u_{0,1}} \frac{\mathcal{D}_{, u_{-1,0}}}{\mathcal{C}_{, u_{-1,0}}}\left(\alpha, u_{0,1}^{\prime}\right) d u_{0,1}^{\prime}+\gamma
$$

where $\alpha$ and $\beta$ are some fixed values of the variables $u_{0,0}$ and $u_{0,1}$ at which the integrals are well defined, while $\gamma$ is an arbitrary integration constant.

\section{$5 \quad$ Examples}

Here we consider the linearizability conditions in the case of some interesting examples.

\subsection{Liouville equation}

Let us consider the discrete Liouville equation [12]

$$
u_{1,1}=\frac{\left(u_{1,0}-1\right)\left(u_{0,1}-1\right)}{u_{0,0}} \doteq F\left(u_{0,0}, u_{0,1}, u_{1,0}\right) \text {. }
$$


In [12] it was shown that the transformation

$$
u_{0,0}=\frac{\tilde{u}_{1,0} \tilde{u}_{0,1}}{\left(\tilde{u}_{1,0}-\tilde{u}_{0,0}\right)\left(\tilde{u}_{0,1}-\tilde{u}_{0,0}\right)},
$$

maps solutions of the linear equation

$$
\tilde{u}_{0,0}-\tilde{u}_{1,0}-\tilde{u}_{0,1}+\tilde{u}_{1,1}=0
$$

into solutions of (5.1). This transformation is not of the form considered here as depends on three points. However this example might also be linearized by the transformations considered in the previous sections.

We can try to linearize this discrete Liouville equation by a point transformation. The necessary conditions $(2.9),(2.10)$ are identically satisfied and the linearizing point transformation, obtained by integrating (2.6), is given by

$$
f\left(u_{0,0}\right)=A\left[B+\log \left(u_{0,0}-1\right)\right]
$$

where $A \neq 0$ and $B$ are arbitrary constants. One can easily see that it does not exist any value of the constants $B, a \neq 0, b \neq 0$ and $c \neq 0$ for which the function $f\left(u_{0,0}\right)$ satisfies (2.4) identically modulo (5.1) (the multiplicative constant $A$ is inessential as it can be always rescaled away). Hence the Liouville equation (5.1) cannot be linearized by a point transformation.

We can try to linearize by a two points transformation of the form (1.4). As $\mathbb{F}=\mathbb{K}=0$ identically, we are in the fourth case. Moreover the two linearizabilty conditions (3.11), (3.20) are identically satisfied and the overdetermined system of differential equations (3.26) reads

$$
\begin{aligned}
& g_{u_{0,0}, u_{0,0}}-\frac{1-u_{0,1}}{u_{0,0}} g_{u_{0,0}, u_{0,1}}+\frac{1}{u_{0,0}} g_{u_{0,0}}=0, \\
& g_{u_{0,1}, u_{0,1}}-\frac{u_{0,0}}{1-u_{0,1}} g_{u_{0,0}, u_{0,1}}-\frac{1}{1-u_{0,1}} g_{u_{0,1}}=0,
\end{aligned}
$$

whose solution is given by

$$
g\left(u_{0,0}, u_{0,1}\right)=\theta(\xi)+C \log \left(u_{0,0}\right)+D, \quad \xi=\frac{u_{0,1}-1}{u_{0,0}},
$$

where $C$ and $D$ are arbitrary constants and $\theta \neq 0$ is an arbitrary function of its arguments. As one can see, the system (5.2) does not specify the two points transformation. To define it we need to introduce (5.3) into (3.5), (3.16). In this way we get a system of two first order differential-difference equations involving $\theta$ and $T_{1} \theta$. From them we can extract a first order ordinary differential equation for $\theta$ which depends on $\xi$ and $u_{1,0}$. As a consequence this equation splits into an overdetermined system of two first order ordinary differential equations for $\theta(\xi)$, whose solution is given by

$$
\theta=C \log (\xi+1)+\alpha, \quad b=-1, \quad a=-c,
$$

where $\alpha$ is an arbitrary constant and $C \neq 0$. Hence, after a reparametrization of $D$,

$$
g\left(u_{0,0}, u_{0,1}\right)=C\left[\log \left(u_{0,0}+u_{0,1}-1\right)+D\right] .
$$

A necessary condition for (5.4) to be a linearizing transformation, is that (3.1) be identically satisfied modulo (5.1). It is easy to show that it is not possible to find a value of $D$ and $C \neq 0$ such that this condition is satisfied. In conclusion the equation (5.1) cannot be linearized by a two points transformation. 
If we consider the linearization through a Hopf-Cole transformation, we are in the case when $\mathbb{F}=\mathbb{K}=0$ and the linearizability conditions $\mathcal{R}_{, u_{1,0}}=\mathcal{U}_{u_{1,0}}=0$ are also satisfied. The equations for the functions $\psi$ and $\phi$ read

$$
\phi_{, u_{0,1}}+\frac{u_{0,0}}{u_{0,1}-1} \phi_{, u_{0,0}}+\frac{1}{u_{0,1}-1}=0, \quad \psi_{, u_{0,-1}}+\frac{u_{0,0}-1}{u_{0,-1}} \psi_{, u_{0,0}}+\frac{1}{u_{0,0}}=0,
$$

and their solution imply

$$
h+1 / b=\sigma(\tilde{\xi}) \rho\left(u_{0,0}\right), \quad \frac{h+1 / b}{h}=\kappa(\tilde{\xi}) \tau\left(u_{0,1}\right), \quad \tilde{\xi} \doteq \frac{u_{0,1}-1}{u_{0,0}},
$$

where $\sigma, \rho, \kappa$ and $\tau$ are arbitrary nonzero functions of their argument. The function $\theta\left(u_{0,0}, u_{0,1}\right)$ defined in (4.17), is specified by the conditions $\mathcal{C}_{, u_{-1,0}}=\mathbb{F}=0$. The necessary conditions $\mathcal{D}_{, u_{-1,0}}=\tilde{\mathcal{T}}_{u_{1,0}}=0$ are satisfied and the two equations (4.18), (4.21) respectively read

$$
\theta_{, u_{0,0}}+\frac{u_{0,1}}{u_{0,0}-1} \theta_{, u_{0,1}}=0, \quad \theta_{, u_{0,1}}+\frac{u_{0,0}}{u_{0,1}-1} \theta_{, u_{0,0}}=0
$$

The only admissible solution of this system is a constant. The solution of the overdetermined system of the two functional equations (5.5) and of the Hopf-like partial differential equation (4.20), after a reparametrization of the constant $\theta$, is given by

$$
h=-\frac{\gamma\left(u_{0,1}-1\right)+\delta}{b \delta+\tilde{\theta} u_{0,0}}
$$

where $\gamma \neq 0, \delta$ and $\tilde{\theta} \neq 0$ are arbitrary constants. A necessary condition to obtain a linearizing transformation is that (4.1) be identically satisfied modulo (5.1). No nonzero value of $a, b, c, \gamma, \tilde{\theta}$ and $\delta$ can satisfy this condition, hence (5.1) cannot be linearized by a Hopf-Cole transformation too.

\subsection{Second Liouville equation}

Let us consider the following version of the discrete Liouville equation

$$
w_{1,1}=\frac{w_{1,0} w_{0,1}-1}{w_{0,0}} \doteq F\left(w_{0,0}, w_{0,1}, w_{1,0}\right) .
$$

As shown in [12], the noninvertible transformation $u_{0,0}=w_{1,0} w_{0,1}$ maps solutions of (5.6) into solutions of (5.1).

Let us look for a linearizing point transformation. The necessary conditions (2.10) are identically satisfied while condition (2.9) reads $-1 / u_{0,0}^{3}=0$. Hence we can conclude that (5.6) cannot be linearized by a point transformation.

Let us look for a linearizing two points transformation. We are in the subcase (1) as $\mathbb{F}=-\mathbb{K}=-1 / u_{0,0}^{3} \neq 0$ and $\mathcal{P}=-1 / u_{0,0} \neq 0$. Moreover we have $\mathcal{M}=-1 / u_{0,0}, \mathcal{N}=\mathcal{Q}=0$. The integrability conditions (3.14), (3.15), (3.23), (3.24) are identically satisfied while the conditions (3.25) cannot be satisfied. As a consequence (5.6) cannot be linearized by a two points transformation.

If we consider the linearization through a Hopf-Cole transformation, we are in the case where $\mathbb{F} \neq 0, \mathbb{K} \neq 0$ and each of the two equations (4.7), (4.8) splits into two equations

$$
\phi_{, u_{0,0}}=\mathcal{S}\left(u_{0,0}, u_{1,0}, u_{0,1}\right) \doteq \frac{\mathcal{R}_{, u_{1,0}} F_{, u_{0,0}}^{2}}{\mathbb{F}}, \quad \phi_{, u_{0,1}}=\mathcal{R}+\frac{F_{, u_{0,1}}}{F_{, u_{0}, 0}} \mathcal{S}
$$




$$
\psi_{, u_{0,0}}=\mathcal{V}\left(u_{0,-1}, u_{0,0}, u_{1,0}\right) \doteq \frac{\mathcal{U}_{, u_{1,0}} K_{, u_{0,0}}^{2}}{\mathbb{K}}, \quad \psi_{, u_{0,-1}}=\mathcal{U}+\frac{K_{, u_{0,-1}}}{K_{, u_{0,0}}} \mathcal{V}
$$

which respectively read

$$
\phi_{, u_{0,0}}+\frac{1}{u_{0,0}}=0, \quad \phi_{, u_{0,1}}=0, \quad \psi_{, u_{0,0}}+\frac{1}{u_{0,0}}=0, \quad \psi F_{, u_{0,-1}}=0 .
$$

The necessary conditions $\left(\mathcal{S}_{,_{1,0}}, \mathcal{V}_{, u_{1,0}}\right)=(0,0)$ are respected and the solutions of the two equations respectively imply

$$
h+\frac{1}{b}=e^{\alpha \frac{u_{0,1}}{u_{0,0}}} \rho\left(u_{0,0}\right), \quad \frac{h+1 / b}{h}=e^{\beta \frac{u_{0,0}}{u_{0,1}}} \tau\left(u_{0,1}\right),
$$

where $\alpha$ and $\beta$ are two arbitrary nonzero constants and $\rho$ and $\tau$ are arbitrary nonzero functions of their arguments. It is not difficult to see that no $\alpha, \beta, \theta$ and $\tau$ exist, giving a nontrivial, e.g. nonconstant, solution for $h$. Hence (5.6) cannot be linearized by a Hopf-Cole transformation too.

\section{$5.3 \mathcal{Q}_{+}$equation linearizable upto $5^{\text {th }}$ order by a multiple scale expansion [3]}

Let us consider the equation

$$
\begin{aligned}
& \zeta u_{0,0} u_{1,0} u_{0,1} u_{1,1}+a_{1}\left(u_{0,0}+u_{1,1}\right)+a_{2}\left(u_{1,0}+u_{0,1}\right)+\gamma_{1} u_{0,0} u_{1,1} \\
& +\frac{a_{2} \gamma_{1}}{a_{1}} u_{1,0} u_{0,1}+\frac{\left(a_{1}+a_{2}\right) \gamma_{1}}{2 a_{1}}\left(u_{0,0} u_{0,1}+u_{1,0} u_{1,1}+u_{0,0} u_{1,0}+u_{0,1} u_{1,1}\right) \\
& +\frac{\left(a_{1}+2 a_{2}\right) \gamma_{1}^{2}}{4 a_{1}^{2}}\left(u_{0,0}+u_{1,1}\right) u_{1,0} u_{0,1}+\frac{\left(2 a_{1}+a_{2}\right) \gamma_{1}^{2}}{4 a_{1}^{2}}\left(u_{1,0}+u_{0,1}\right) u_{0,0} u_{1,1},
\end{aligned}
$$

where $a_{1}, a_{2}, \gamma_{1}, \zeta$ are arbitrary real parameters with $\left|a_{1}\right| \neq\left|a_{2}\right|$ and $a_{j} \neq 0, j=1,2$. In [3] it has been shown that this equation passes a linearizability test based on multiscale analysis up to fifth order in the perturbation parameter for small $u$.

Let us search for the possibility to linearize (5.7) by a point transformation. Of the necessary conditions $(2.10), \mathcal{H}^{(0)}=0$ is automatically satisfied while $\mathcal{K}^{(0)}=0$ and (2.9) can be satisfied if and only if

$$
\zeta=\frac{\left(a_{1}+a_{2}\right) \gamma_{1}^{3}}{4 a_{1}^{3}} .
$$

In this case the linearizing point transformation obtained integrating (2.6) is given by

$$
f\left(u_{0,0}\right)=A\left[\frac{1}{2 a_{1}+\gamma_{1} u_{0,0}}+B\right],
$$

where $A \neq 0$ and $B$ are constants. Inserting $f\left(u_{0,0}\right)$ into (2.4), one finds that this relation is identically satisfied modulo (5.7), (5.8) when $c=b a_{1} / a_{2}$. Finally, inserting $f\left(u_{0,0}\right)$ and $c$ into (2.1), it is straightforward to see that this relation results identically satisfied modulo (5.7), (5.8) when $B=-1 /\left(2 a_{1}\right), c=1$ and $a=b=a_{2} / a_{1}$. Equation (5.9) is the linearizing point transformation when $B=-1 /\left(2 a_{1}\right)$.

Let us consider the case $\zeta \neq\left(a_{1}+a_{2}\right) \gamma_{1}^{3} /\left(4 a_{1}^{3}\right)$. If we search for a linearizing two points transformation, as $a_{j} \neq 0, j=1,2$, we are always in the subcase $(a)$. Then the necessary condition (3.14) cannot be satisfied. So, if the condition (5.8) is not satisfied, (5.7) cannot be linearized by a two points transformation.

We can try to linearize (5.7) by a Hopf-Cole transformation. We are in the case where $\mathcal{F} \neq 0$ and the equation (4.7) splits into two equations. As the necessary condition $\mathcal{S}_{, u_{1,0}}=0$ cannot be satisfied, (5.7) cannot be linearized by a Hopf-Cole transformation. We can conclude that, if the condition (5.8) is not satisfied, (5.7) cannot be linearized by neither a point, nor contact or Hopf-Cole transformation. 


\subsection{Hietarinta equation}

Let us consider the Hietarinta equation $[4,11]$

$$
\frac{u_{0,0}+e_{2}}{u_{0,0}+e_{1}} \frac{u_{1,1}+o_{2}}{u_{1,1}+o_{1}}=\frac{u_{1,0}+e_{2}}{u_{1,0}+o_{1}} \frac{u_{0,1}+o_{2}}{u_{0,1}+e_{1}}
$$

where $e_{j}$ and $o_{j}, j=1,2$ are arbitrary parameters.

\subsubsection{Linearizing one point transformation}

The necessary conditions (2.9), (2.10) of linearizability through a point transformation are identically satisfied and the integration of equation (2.6) gives

$$
f\left(u_{0,0}\right)=A\left[\log \left(\frac{u_{0,0}+o_{1}}{u_{0,0}+o_{2}}\right)+B\right]
$$

where $A \neq 0$ and $B$ are arbitrary constants. One can easily see that no values of the constants $B$, $a \neq 0, b \neq 0$ and $c \neq 0$ exist for which the function $f\left(u_{0,0}\right)$ satisfies (2.4) identically modulo the Hietarinta equation. As a consequence (5.10) cannot be linearized by a point transformation.

\subsubsection{Linearizing two points transformation}

As $\mathbb{F}=\mathbb{K}=0$, we are in the case (4). Moreover the two linearizability conditions (3.11), (3.20) are identically satisfied and the overdetermined system of differential equations (3.26) reads

$$
\begin{aligned}
& g_{, u_{0,0}, u_{0,0}}+\frac{\left(e_{2}-e_{1}\right)\left(u_{0,1}+o_{1}\right)\left(u_{0,1}+o_{2}\right)}{\left(o_{2}-o_{1}\right)\left(u_{0,0}+e_{1}\right)\left(u_{0,0}+e_{2}\right)} g_{, u_{0,0}, u_{0,1}}+\frac{2 u_{0,0}+e_{1}+e_{2}}{\left(u_{0,0}+e_{1}\right)\left(u_{0,0}+e_{2}\right)} g_{, u_{0,0}}=0, \\
& g_{, u_{0,1}, u_{0,1}}+\frac{\left(o_{2}-o_{1}\right)\left(u_{0,0}+e_{1}\right)\left(u_{0,0}+e_{2}\right)}{\left(e_{2}-e_{1}\right)\left(u_{0,1}+o_{1}\right)\left(u_{0,1}+o_{2}\right)} g_{, u_{0,0}, u_{0,1}}+\frac{2 u_{0,1}+o_{1}+o_{2}}{\left(u_{0,1}+o_{1}\right)\left(u_{0,1}+o_{2}\right)} g_{0,0, u_{0,1}}=0 .
\end{aligned}
$$

The solution of (5.11) is given by

$$
g\left(u_{0,0}, u_{0,1}\right)=\theta(\xi)+A \log \left(\frac{u_{0,1}+o_{2}}{u_{0,1}+o_{1}}\right)+B, \quad \xi=\frac{\left(u_{0,0}+e_{2}\right)\left(u_{0,1}+o_{1}\right)}{\left(u_{0,0}+e_{1}\right)\left(u_{0,1}+o_{2}\right)},
$$

where $A$ and $B$ are arbitrary constants and $\theta \neq 0$ is an arbitrary function of its argument. As one can see, the system (5.11) is not sufficient to specify the eventual two points transformation. We need to introduce (5.12) into (3.5), (3.16). In this way we get a system of first order differential equations involving $\theta$ and $T_{1} \theta$. From them we can extract a first order ordinary differential equation for $\theta$ which depends on $\xi, u_{0,0}$ and $u_{0,1}$. As a consequence this equation splits into an overdetermined system of four ordinary differential equations for $\theta(\xi)$. This system has no solution for generic $e_{j}, o_{j}, j=1,2$. As a consequence the Hietarinta equation cannot be linearized by a two points transformation.

\subsubsection{Linearizing one point Hopf-Cole transformation}

We are in the case when (2.9), (2.10) are satisfied, and the integration of (4.4) gives

$$
h\left(u_{0,0}\right)=\frac{1}{b}\left[A\left(\frac{u_{0,0}+e_{1}}{u_{0,0}+o_{2}}\right)^{B}-1\right],
$$

where $A \neq 0$ and $B \neq 0$ are arbitrary integration constants. We have that (4.3) can be identically satisfied modulo the Hietarinta equation if and only if

$$
A=\left(1-\frac{a b}{c}\right)\left(\frac{o_{2}-o_{1}}{e_{1}-o_{1}}\right), \quad B=1
$$


Finally (4.2) is identically satisfied modulo the Hietarinta equation if and only if

$$
A=-\frac{o_{2}-e_{2}}{e_{2}-e_{1}}, \quad a=\frac{c\left(e_{2}-o_{1}\right)\left(o_{2}-e_{1}\right)}{b\left(e_{2}-e_{1}\right)\left(o_{2}-o_{1}\right)},
$$

so that

$$
\tilde{u}_{0,1}=-\frac{1}{b} \frac{\left(o_{2}-e_{1}\right)\left(u_{0,0}+e_{2}\right)}{\left(e_{2}-e_{1}\right)\left(u_{0,0}+o_{2}\right)} \tilde{u}_{0,0} .
$$

Through the gauge transformation $\tilde{u}_{0,0} \doteq(-b / c)^{n}(-b)^{-m} w_{0,0}$ we get a simplified linearizing transformation

$$
\begin{aligned}
& w_{0,1}=\frac{\left(e_{1}-o_{2}\right)\left(u_{0,0}+e_{2}\right)}{\left(e_{1}-e_{2}\right)\left(u_{0,0}+o_{2}\right)} w_{0,0}, \\
& w_{0,0}+a w_{1,0}-w_{0,1}+w_{1,1}=0, \quad a=-\frac{\left(o_{1}-e_{2}\right)\left(e_{1}-o_{2}\right)}{\left(e_{1}-e_{2}\right)\left(o_{1}-o_{2}\right)} .
\end{aligned}
$$

Is is moreover straightforward to demonstrate that if (5.13), (5.14) are satisfied, then also the Hietarinta equation is satisfied.

\subsubsection{Linearizing two point Hopf-Cole transformation [11]}

We are in the case defined by the conditions $\mathcal{C}_{, u_{-1,0}}=\mathcal{D}_{, u_{-1,0}}=\mathcal{T}_{u_{1,0}}=\mathbb{F}=0$ and thus the linearizing function is defined by the equations (4.18), (4.21) which read

$$
\begin{aligned}
& \theta_{, u_{0,0}}+\frac{\left(e_{2}-o_{1}\right)\left(u_{0,1}+o_{1}\right)\left(u_{0,1}+o_{2}\right)}{\left(o_{2}-o_{1}\right)\left(u_{0,0}+o_{1}\right)\left(u_{0,0}+e_{2}\right)} \theta_{, u_{0,1}}=2 \frac{u_{0,0}\left(o_{2}-o_{1}\right)-u_{0,1}\left(e_{2}-o_{1}\right)+o_{1}\left(o_{2}-e_{2}\right)}{\left(o_{2}-o_{1}\right)\left(u_{0,0}+o_{1}\right)\left(u_{0,0}+e_{2}\right)}, \\
& \theta_{, u_{0,1}}+\frac{\left(o_{2}-e_{1}\right)\left(u_{0,0}+e_{1}\right)\left(u_{0,0}+e_{2}\right)}{\left(e_{2}-e_{1}\right)\left(u_{0,1}+e_{1}\right)\left(u_{0,1}+o_{2}\right)} \theta_{, u_{0,0}}=2 \frac{u_{0,0}\left(o_{2}-e_{1}\right)-u_{0,1}\left(e_{2}-e_{1}\right)+e_{1}\left(o_{2}-e_{2}\right)}{\left(e_{2}-e_{1}\right)\left(u_{0,1}+e_{1}\right)\left(u_{0,1}+o_{2}\right)},
\end{aligned}
$$

whose solution is given by

$$
\theta\left(u_{0,0}, u_{0,1}\right)=\log \left[\left(\frac{u_{0,0}+e_{2}}{u_{0,1}+o_{2}}\right)^{2}\right]+\alpha,
$$

where $\alpha$ is an arbitrary integration constant. Then we can solve the Hopf-like equation (4.20) by separation of variables, $h=A\left(u_{0,0}\right) B\left(u_{0,1}\right)$, obtaining

$$
h\left(u_{0,0}, u_{0,1}\right)=\frac{e^{\alpha}\left(u_{0,0}+e_{2}\right)}{\delta\left(u_{0,0}+e_{2}\right)+\beta} \frac{\gamma\left(u_{0,1}+o_{2}\right)-\beta}{u_{0,1}+o_{2}},
$$

where $\beta, \gamma$ and $\delta$ are arbitrary integration constants. A necessary condition to obtain the linearization is that (4.1) be identically satisfied for all $u_{0,0}, u_{1,0}, u_{0,1}, u_{0,2}, e_{j}, o_{j}, j=1,2$ modulo the Hietarinta equation, from which we get

$$
e^{\alpha}=\frac{a \beta\left(o_{2}-o_{1}\right)}{c\left(e_{2}-o_{1}\right)\left[\beta+\gamma\left(e_{2}-o_{2}\right)\right]}, \quad \delta=-\frac{\beta \gamma}{\beta+\gamma\left(e_{2}-o_{2}\right)}, \quad b=\frac{c\left(o_{2}-e_{1}\right)\left(e_{2}-o_{1}\right)}{a\left(e_{2}-e_{1}\right)\left(o_{2}-o_{1}\right)} .
$$

When we insert the obtained values of $e^{\alpha}, \delta$ and $b$ into the transformation (5.15), the two equations for $\psi$ and $\phi$ are identically satisfied. As $h$ depends on $u_{0,0}$ and $u_{0,1}$, it is necessary that $\beta \neq 0$. By redefining $\gamma \doteq \beta \epsilon$ we can eliminate the parameter $\beta$ from the transformation. The transformation as well as the coefficient $b$ of the linear equation so far obtained depend in a multiplicative way from the ratio $a / c$. Hence, performing the transformation $\tilde{u}_{n, m} \doteq$ 
$\chi^{m} v_{n, m}$, where $\chi$ is a constant, the linear equation (1.2) and the Hopf-Cole transformation (1.4) respectively read

$$
\begin{aligned}
& v_{n, m}+a v_{n+1, m}+b \chi v_{n, m+1}+c \chi v_{n+1, m+1}=0, \\
& v_{n, m+1}=\frac{1}{\chi} h\left(u_{n, m}, u_{n, m+1}\right) v_{n, m},
\end{aligned}
$$

and choosing $\chi=-a / c$ we can remove the ratio $a / c$ from the expressions of $h$ and $b$. In other words we can always choose a "gauge" for the linearizing transformation in which $a=-c$. In conclusion we have

$$
h=\frac{\left(o_{2}-o_{1}\right)\left(u_{0,0}+e_{2}\right)\left[1-\epsilon\left(u_{0,1}+o_{2}\right)\right]}{\left(e_{2}-o_{1}\right)\left[1-\epsilon\left(u_{0,0}+o_{2}\right)\right]\left(u_{0,1}+o_{2}\right)}, \quad b=-\frac{\left(o_{2}-e_{1}\right)\left(e_{2}-o_{1}\right)}{\left(e_{2}-e_{1}\right)\left(o_{2}-o_{1}\right)} .
$$

\subsubsection{Inverse two point Hopf-Cole transformation}

To be able to solve the Hietarinta equation explicitly we look here for the inverse formula, i.e. the formula which provide the solution of the Hietarinta equation in terms of those of the linear equation (1.2).

Given a solution of the Hietarinta equation, let us perform a Hopf-Cole transformation (1.5) with $h$ given in (5.16). Extracting from the Hopf-Cole transformation $u_{0,1}$ as a function of $u_{0,0}$ and $\eta_{0,0} \doteq \tilde{u}_{0,1} / \tilde{u}_{0,0}$ and inserting it, together with its consequences, in the Hietarinta equation, if $\epsilon \neq \frac{1}{\left(o_{2}-o_{1}\right)}$, we obtain $u_{1,0}$ as a function of $u_{0,0}, \eta_{0,0}$ and $\eta_{1,0}$. The compatibility between the functions $u_{0,1}\left(u_{0,0}, \eta_{0,0}\right)$ and $u_{1,0}\left(u_{0,0}, \eta_{0,0}, \eta_{1,0}\right)$ gives a second degree polynomial equation in $u_{0,0}$ which must be satisfied for all $u_{0,0}$. Taking into account that the ratio $\eta_{0,0}$ cannot in general be a constant when $u_{0,0}$ satisfies the Hietarinta equation, we have that, if $\epsilon \neq \frac{1}{\left(o_{2}-o_{1}\right)}$, $\tilde{u}_{0,0}$ will satisfy the linear and in general nonautonomous equation

$$
\left(1-\alpha_{n}\right) \tilde{u}_{0,0}-c \tilde{u}_{1,0}+b\left(1-\alpha_{n}\right) \tilde{u}_{0,1}+c \tilde{u}_{1,1}=0, \quad c=\frac{\left(e_{1}-o_{1}\right)\left(e_{2}-o_{1}\right)}{\left(e_{2}-e_{1}\right)\left(o_{2}-o_{1}\right)},
$$

where $\alpha_{n}$ is an $n$-dependent integration function depending on the initial values $u_{n, 0}$ and $\tilde{u}_{n, 0}$ given by

$$
\alpha_{n}=1-\frac{\left(e_{1}-o_{1}\right)\left[1-\epsilon\left(u_{n, 0}+o_{2}\right)\right]\left(u_{n+1,0}+o_{1}\right) \tilde{u}_{n+1,0}}{\left(o_{2}-o_{1}\right)\left(u_{n, 0}+e_{1}\right)\left[1-\epsilon\left(u_{n+1,0}+o_{2}\right)\right] \tilde{u}_{n, 0}} .
$$

When $\alpha_{n} \neq 1$, performing the "gauge" transformation $\tilde{u}_{n, m} \doteq \tau_{n} v_{n, m}$, with $\tau_{n+1}=\left(1+\alpha_{n}\right) \tau_{n}$, the Hopf-Cole transformation is invariant while the function $v_{n, m}$ will satisfy the linear autonomous equation

$$
v_{0,0}-c v_{1,0}+b v_{0,1}+c v_{1,1}=0 .
$$

When $\epsilon=\frac{1}{\left(o_{2}-o_{1}\right)}$, the function $h$ becomes

$$
h=\frac{\left(o_{2}-o_{1}\right)\left(u_{0,0}+e_{2}\right)\left(u_{0,1}+o_{1}\right)}{\left(e_{2}-o_{1}\right)\left(u_{0,0}+e_{1}\right)\left(u_{0,1}+o_{2}\right)} .
$$

Inserting the corresponding Hopf-Cole transformation into the Hietarinta equation we get $u_{0,0}$ in terms of $\eta_{0,0}$ and $\eta_{1,0}$

$$
u_{0,0}=-\frac{e_{2}\left(e_{1}-o_{1}\right)\left(o_{2}-o_{1}\right)+o_{1}\left(e_{2}-o_{1}\right)\left(o_{2}-e_{1}\right) \eta_{0,0}-e_{1}\left(e_{2}-o_{1}\right)\left(o_{2}-o_{1}\right) \eta_{1,0}}{\left(e_{1}-o_{1}\right)\left(o_{2}-o_{1}\right)+\left(e_{2}-o_{1}\right)\left(o_{2}-e_{1}\right) \eta_{0,0}-\left(e_{2}-o_{1}\right)\left(o_{2}-o_{1}\right) \eta_{1,0}}
$$


and its insertion with its consequences into $u_{0,1}\left(u_{0,0}, \eta_{0,0}\right)$ implies for the function $\tilde{u}_{0,0}$ the same evolution (5.17) with $\alpha_{n}$ given by the limit as $\epsilon \rightarrow \frac{1}{\left(o_{2}-o_{1}\right)}$ of (5.18) and hence (5.19) for $v_{0,0}$, just as was shown in [11]. As a consequence $u_{0,0}$, given by (5.21), becomes

$$
u_{0,0}=-\frac{e_{1}\left(o_{2}-o_{1}\right) v_{0,0}-o_{1}\left(e_{1}-o_{1}\right) v_{1,0}}{\left(o_{2}-o_{1}\right) v_{0,0}-\left(e_{1}-o_{1}\right) v_{1,0}}
$$

Conversely, if $v_{0,0}$ satisfies (5.19), then it is possible to demonstrate that $u_{0,0}$ given by $(5.22)$ satisfies the Hietarinta equation.

In the relations $(5.21),(5.22)$ the role of the variables $u_{n, m}$ and $v_{n, m}$ appears inverted with respect to those given by the Hopf-Cole transformation (1.5), (1.6).

The inverted transformation, consequently, appears only when $\epsilon=\frac{1}{\left(o_{2}-o_{1}\right)}$. The relation (5.22) is the inverse in the space of the solutions of the Hietarinta equation of the relation (1.6) with the function $k$ defined in (1.7) corresponding to an $h$ as given in (5.20) and restricting the result to the space of the solutions of the Hietarinta equation, that is

$$
\frac{v_{1,0}}{v_{0,0}}=k=\frac{\left(o_{2}-o_{1}\right)\left(u_{0,0}+e_{1}\right)}{\left(e_{1}-o_{1}\right)\left(u_{0,0}+o_{1}\right)} .
$$

Finally, inserting the initial value at $m=0$ of (5.23) into (5.18), we get $\alpha_{n}=0$. By the transformation $v_{0,0} \doteq c^{-n} w_{0,0}$, we can simplify further (5.19) which together with (5.23) becomes

$$
\tilde{w}_{0,0}-\tilde{w}_{1,0}+b \tilde{w}_{0,1}+\tilde{w}_{1,1}=0, \quad \tilde{w}_{1,0}=\frac{\left(e_{2}-o_{1}\right)\left(u_{0,0}+e_{1}\right)}{\left(e_{2}-e_{1}\right)\left(u_{0,0}+o_{1}\right)} \tilde{w}_{0,0} .
$$

The second relation in (5.24) represents another linearizing one point Hopf-Cole transformation $\tilde{w}_{1,0}=\tilde{h}\left(u_{0,0}\right) \tilde{w}_{0,0}$.

\subsubsection{Solution of the Hietarinta equation}

The general integral of the Hietarinta equation is obtained inserting in the inverse of the second relation (5.24) the solution of the initial-boundary value problem for the linear equation given in (5.24). This solution is given by

- if $b=-1$ by $\tilde{w}_{n, m}=\tilde{w}_{n, 0}+\tilde{w}_{0, m}-\tilde{w}_{0,0}$,

- if $b \neq-1$ by $\tilde{w}_{n, m}=\frac{1}{2 \pi \mathrm{i}} \oint_{C} \zeta_{m}(z) z^{n-1} d z$, where

$$
(1-z) \zeta_{m}+(b+z) \zeta_{m+1}=z\left(\tilde{w}_{0, m+1}-\tilde{w}_{0, m}\right), \quad \zeta_{0}(z)=\sum_{j=0}^{+\infty} \tilde{w}_{j, 0} z^{-j}
$$

and $C$ represents a counterclockwise circumference in the complex $z$-plane, centered in $z=0$ and lying inside the region of convergence of the series $\sum_{j=0}^{+\infty} \tilde{w}_{j, m} z^{-j}$.

For example, when $b \neq-1$, an explicit solution in the plane $n \geq 0$ of the initial-boundary value problem characterized by $\tilde{w}_{0, m}=1, \tilde{w}_{n, 0}=2^{-n}, n \geq 0\left(\tilde{w}_{n, 0}=0, n<0\right)$, so that $\zeta_{0}=2 z /(2 z-1),|z|>1 / 2$, is given, if $b=-\frac{1}{2}$, by

$$
\begin{aligned}
\tilde{w}_{n, m} & =\sum_{\rho=\max \{n, m\}}^{n+m}\left(\begin{array}{c}
m \\
\rho-n
\end{array}\right)\left(\begin{array}{c}
\rho \\
m
\end{array}\right) \frac{(-1)^{n+m-\rho}}{2^{\rho-m}}, \quad m \geq 0, \\
\tilde{w}_{n, m} & =\sum_{\rho=\max \{n,|m|-1\}}^{n+|m|-1}\left(\begin{array}{c}
|m|-1 \\
\rho-n
\end{array}\right)\left(\begin{array}{c}
\rho \\
|m|-1
\end{array}\right)\left(-\frac{1}{2}\right)^{n+|m|-\rho-1}, \quad m \leq-1,
\end{aligned}
$$


and, if $b \neq-\frac{1}{2}$, by

$$
\begin{aligned}
& \tilde{w}_{n, m}=\frac{1}{2^{n}(-1-2 b)^{m}}-\sum_{\kappa=0}^{m-1} \sum_{\rho=\max \{n, \kappa\}}^{n+m}\left(\begin{array}{c}
m \\
\rho-n
\end{array}\right)\left(\begin{array}{c}
\rho \\
\kappa
\end{array}\right) \frac{(-1)^{n+m-\kappa} b^{\rho-\kappa}}{(b+1 / 2)^{m-\kappa}}, \quad m \geq 1, \\
& \tilde{w}_{n, m}=\frac{(-1-2 b)^{|m|}}{2^{n}}-\sum_{\kappa=0}^{|m|-1} \sum_{\rho=\max \{n, \kappa\}}^{n+|m|}\left(\begin{array}{c}
|m| \\
\rho-n
\end{array}\right)\left(\begin{array}{c}
\rho \\
\kappa
\end{array}\right)(-2)^{|m|-\kappa} b^{n+|m|-\rho}, \quad m \leq-1 .
\end{aligned}
$$

\subsection{A nonlinear quad-graph equation linearizable by two point transformation}

As a further example let us consider the simple multilinear equation

$$
u_{0,0}+\alpha u_{1,0}+\epsilon\left[u_{0,0} u_{0,1}+\alpha u_{1,0} u_{1,1}\right]=0 .
$$

As in the case of the discrete Liouville equation the necessary conditions for the linearizability by point transformations are identically satisfied and the linearizing point transformation, obtained by integrating (2.6), is given by

$$
f\left(u_{0,0}\right)=A\left[B+\log \left(u_{0,0}+\frac{1}{\epsilon}\right)\right]
$$

where $A \neq 0$ and $B$ are arbitrary constants. Inserting $f\left(u_{0,0}\right)$ into (2.4), one can easily see that this relation can be identically satisfied modulo (5.25) only if $c=-b$. Differentiating (2.1) with respect to $u_{1,0}$ and inserting $f\left(u_{0,0}\right)$ and $c=-b$ into the resulting relation, we have that no values of the constants $B, a \neq 0, b \neq 0$ and $c \neq 0$ exist for which this can be satisfied identically modulo (5.25). Hence this equation (5.25) cannot be linearized by a point transformation.

We can try to linearize it by a two points transformation of the form (1.4). As $\mathbb{F}=\mathbb{K}=0$ identically, we are in the fourth case. Moreover the two linearizabilty conditions (3.11), (3.20) are identically satisfied and the overdetermined system of differential equations (3.26), (3.27) reads

$$
\begin{aligned}
& g_{u_{0,0}, u_{0,0}}-\frac{1+\epsilon u_{0,1}}{\epsilon u_{0,0}} g_{u_{0,0}, u_{0,1}}+\frac{1}{u_{0,0}} g_{u_{0,0}}=0 \\
& g_{u_{0,1}, u_{0,1}}-\frac{\epsilon u_{0,0}}{1+u \epsilon_{0,1}} g_{u_{0,0}, u_{0,1}}+\frac{\epsilon}{1+\epsilon u_{0,1}} g_{u_{0,1}}=0
\end{aligned}
$$

whose solution is given by

$$
g\left(u_{0,0}, u_{0,1}\right)=\theta(\xi)+C \log \left(u_{0,0}\right)+D, \quad \xi=u_{0,0}\left(u_{0,1}+\frac{1}{\epsilon}\right),
$$

where $C$ and $D$ are arbitrary constants and $\theta \neq 0$ is an arbitrary function of its arguments. Introducing (5.26) in (3.5), (3.16) we get $b=\frac{c}{\alpha}$. The final determining equation (3.2) implies $C=D=0, a=\alpha$ and $\theta=k u_{0,0}\left(1+\epsilon u_{0,1}\right)$. So, in conclusion (5.25) is linearizable by the transformation

$$
g\left(u_{0,0}, u_{0,1}\right)=u_{0,0}\left(1+\epsilon u_{0,1}\right)
$$

into the linear equation

$$
\tilde{u}_{0,0}+\alpha \tilde{u}_{1,0}+b \tilde{u}_{0,1}+\alpha b \tilde{u}_{1,1}=0 .
$$




\subsection{Four $Q R T$-type linearizable equations}

As a final example we consider, as suggested by one of the referees, the four $Q R T$-type linearizable nonlinear partial difference equations recently presented in [13]

$$
\begin{aligned}
& u_{1,1}=\frac{u_{1,0}+u_{0,1}-\left(1-u_{1,0} u_{0,1}\right) u_{0,0}}{1-u_{1,0} u_{0,1}+\left(u_{1,0}+u_{0,1}\right) u_{0,0}}, \\
& u_{1,1}=\frac{u_{1,0}-u_{0,1}+\left(1+u_{1,0} u_{0,1}\right) u_{0,0}}{1+u_{1,0} u_{0,1}-\left(u_{1,0}-u_{0,1}\right) u_{0,0}}, \\
& u_{1,1}=\frac{f_{2}(n, m)+f_{1}(n, m) u_{0,0}}{f_{1}(n, m)-f_{2}(n, m) u_{0,0}}, \\
& f_{1}(n, m) \doteq\left(1+u_{1,0} u_{0,1}\right)\left(u_{1,0}^{2} u_{0,1}^{2}-3 u_{1,0}^{2}-3 u_{0,1}^{2}+8 u_{1,0} u_{0,1}+1\right), \\
& f_{2}(n, m) \doteq\left(u_{0,1}-u_{1,0}\right)\left(3 u_{1,0}^{2} u_{0,1}^{2}-u_{1,0}^{2}-u_{0,1}^{2}+8 u_{1,0} u_{0,1}+3\right), \\
& u_{1,1}=\frac{2 u_{1,0}-u_{0,1}+u_{0,1} u_{1,0}^{2}-\left(1+2 u_{1,0} u_{0,1}-u_{1,0}^{2}\right) u_{0,0}}{1+2 u_{1,0} u_{0,1}-u_{1,0}^{2}+\left(2 u_{1,0}-u_{0,1}+u_{0,1} u_{1,0}^{2}\right) u_{0,0}} .
\end{aligned}
$$

All these equations are linearizable through the transformation $v_{0,0}=\arctan \left(u_{0,0}\right)$ and give the following linear equations

$$
\begin{aligned}
& v_{0,0}-v_{1,0}-v_{0,1}+v_{1,1}=p \pi, \\
& v_{0,0}+v_{1,0}-v_{0,1}-v_{1,1}=p \pi, \\
& v_{0,0}-3 v_{1,0}+3 v_{0,1}-v_{1,1}=p \pi, \\
& v_{0,0}-2 v_{1,0}+v_{0,1}+v_{1,1}=p \pi,
\end{aligned}
$$

where $p \in \mathcal{Z}$. The $p$-dependent right-hand side is a consequence of the multi-valuedness of the arctan function. We can choose $p=0$ if we choose the principle branch of the arctan function, i.e. $\arctan (0)=0$.

Applying the formulas contained in Section 2 it is immediate to show that the necessary conditions $(2.9),(2.10)$ of linearizability through a point transformation are identically satisfied. The integration of equation (2.6) gives

$$
f\left(u_{0,0}\right)=A\left[\arctan \left(u_{0,0}\right)+B\right],
$$

where $A \neq 0$ and $B$ are arbitrary constants. The differential consequences of equation (2.1) (which in this case will have a constant right-hand side not necessarily equal to zero) can be identically satisfied modulo the QRT-type equations if and only if

$$
\begin{array}{lll}
a=-1, & b=-1, & c=1, \\
a=1, & b=-1, & c=-1, \\
a=-3, & b=3, & c=-1, \\
a=-2, & b=1, & c=1 .
\end{array}
$$

With these values of the coefficients $(a, b, c),(2.1)$ is identically satisfied modulo the corresponding equations. In the first three cases its right hand side is equal to $p \pi$ for arbitrary $B$ while in the fourth case it is equal to $B+p \pi$. As $B$ is arbitrary, for the sake of simplicity we will choose $B=0$ in all cases.

\section{Conclusions}

In this paper we have considered the possibility of linearizing nonlinear partial difference equations defined on a quad-graph by the use of point one, two points and a Hopf-Cole transformation. Imposing the existence of such transformations we obtained for the equations some 
necessary linearizability conditions and some differential equations for the transformations. We have applied our results to some nonlinear partial difference equations, some of which already known to be linearizable, to test our procedure. In the case of the nonlinear equation (5.7), obtained as a result of a classification of multilinear equations on the quad-graph by the multiple scales expansion up to fifth order, we have been able to show that all linearizability conditions considered here imply the constraint on the coefficients (5.8).

In the case of the Hietarinta equation we have been able to find out a new linearizing HopfCole transformation depending just on one function, up to our knowledge unknown.

In the verification of the examples presented in [13], we discovered an accidental misprint in (5.27), as in the original article the signs of $v_{1,0}$ and $v_{0,1}$ are inverted.

A few problems are still open. We have just considered here the case of one point, two points and two points Hopf-Cole transformations but it could be interesting to consider in the future more general cases, maybe combining it with results on the integrability of the nonlinear equations. Moreover one would like to include nonautonomous transformations and lattice dependent linear equations.

\section{Acknowledgments}

LD and SC have been partly supported by the Italian Ministry of Education and Research, PRIN "Continuous and discrete nonlinear integrable evolutions: from water waves to symplectic maps" from 2010.

\section{References}

[1] Abel N.H., Methode generale pour trouver des fonctions d'une seule quantite variable lorsqu'une propriete de ces fonctions est exprimee par une equation entre deux variables, Mag. Naturvidenskab. 1 (1823), 1-10, reproduced in Ouvres Completes, Vol. I, Christiania, 1881, 1-10.

Aczel J., Lectures on functional equations and their applications, Mathematics in Science and Engineering, Vol. 19, Academic Press, New York - London, 1966.

[2] Cole J.D., On a quasi-linear parabolic equation occurring in aerodynamics, Quart. Appl. Math. 9 (1951), $225-236$.

[3] Hernandez Heredero R., Levi D., Scimiterna C., A discrete linearizability test based on multiscale analysis, J. Phys. A: Math. Theor. 43 (2010), 502002, 14 pages, arXiv:1011.0141.

[4] Hietarinta J., A new two-dimensional lattice model that is 'consistent around a cube', J. Phys. A: Math. Gen. 37 (2004), L67-L73, nlin.SI/0311034.

[5] Hopf E., The partial differential equation $u_{t}+u u_{x}=u_{x x}$, Comm. Pure Appl. Math. 3 (1950), 201-230.

[6] Levi D., Ragnisco O., Bruschi M., Continuous and discrete matrix Burgers hierarchies, Nuovo Cimento B 74 (1983), 33-51.

[7] Levi D., Yamilov R.I., The generalized symmetry method for discrete equations, J. Phys. A: Math. Theor. 42 (2009), 454012, 18 pages, arXiv:0902.4421.

[8] Levi D., Yamilov R.I., Generalized symmetry integrability test for discrete equations on the square lattice, J. Phys. A: Math. Theor. 44 (2011), 145207, 22 pages, arXiv:1011.0070.

[9] Mikhailov A.V., Wang J.P., Xenitidis P., Recursion operators, conservation laws and integrability conditions for difference equations, Theoret. and Math. Phys. 167 (2011), 421-443, arXiv:1004.5346.

[10] Miura R.M., Korteweg-de Vries equation and generalizations. I. A remarkable explicit nonlinear transformation, J. Math. Phys. 9 (1968), 1202-1204.

Miura R.M., Gardner C.S., Kruskal M.D., Korteweg-de Vries equation and generalizations. II. Existence of conservation laws and constants of motion, J. Math. Phys. 9 (1968), 1204-1209.

[11] Ramani A., Joshi N., Grammaticos B., Tamizhmani T., Deconstructing an integrable lattice equation, J. Phys. A: Math. Gen. 39 (2006), L145-L149.

[12] Startsev S.Ya., On non-point invertible transformations of difference and differential-difference equations, SIGMA 6 (2010), 092, 14 pages, arXiv:1010.0361.

[13] Uma Maheswari C., Sahadevan R., On the conservation laws for nonlinear partial difference equations, J. Phys. A: Math. Theor. 44 (2011), 275203, 16 pages. 